\title{
A New Triangular Spectral Element Method II: Mixed Formulation and $h p$-Error Estimates
}

\author{
Bingzhen Zhou ${ }^{1}$, Bo Wang ${ }^{1, *}$, Li-Lian Wang ${ }^{2}$ and Ziqing Xie ${ }^{1}$ \\ ${ }^{1}$ Key Laboratory of High Performance Computing and Stochastic Information \\ Processing (HPCSIP), College of Mathematics and Statistics, Hunan Normal \\ University, Changsha 410081, P. R. China. \\ ${ }^{2}$ Division of Mathematical Sciences, School of Physical and Mathematical \\ Sciences, Nanyang Technological University, 637371, Singapore.
}

Received 14 March 2018; Accepted (in revised version) 15 July 2018

\begin{abstract}
Mixed triangular spectral element method using nodal basis on unstructured meshes is investigated in this paper. The method is based on equivalent first order system of the elliptic problem and rectangle-triangle transforms. It fully enjoys the tensorial structure and flexibility in handling complex domains by using nodal basis and unstructured triangular mesh. Different from the usual Galerkin formulation, the mixed form is particularly advantageous in this context, since it can avoid the singularity induced by the rectangle-triangle transform in the calculation of the matrices, and does not require the evaluation of the stiffness matrix. An $h p$ a priori error estimate is presented for the proposed method. The implementation details and some numerical examples are provided to validate the accuracy and flexibility of the method.
\end{abstract}

AMS subject classifications: 65N30, 65N35, 65N22, 35J25

Key words: Triangular spectral element method, $h p$ error analysis, mixed form, interpolation error in $H^{1}$-norm.

\section{Introduction}

The spectral element method (SEM) (or $h p$ finite element method) [22] integrates the unparalleled accuracy of a spectral method and the geometric flexibility of a finite element method, and also enjoys a high-level parallel computer architecture. As such, it plays an exceedingly important part in large-scale simulations $[4,8,13,14]$. For a long time, we saw SEM through building blocks of quadrilaterals and hexahedra with tensorial structures (QSEM) $[4,8,22]$. The use of tensorial nodal basis functions in a QSEM substantially facilitates both the implementation (e.g., the imposition of continuity across elements) and analysis, as many numerical tools and analysis arguments in one dimension can be

\footnotetext{
${ }^{*}$ Corresponding author. Email addresses: zbzhen@smail.hunnu.edu.cn (B. Z. Zhou), bowang@hunnu.ed u.cn (B. Wang), lilian@ntu.edu.sg (L. L. Wang), ziqingxie@hunnu.edu.cn (Z. Q. Xie)
} 
directly transplanted to multiple dimensions. However, QSEM usually requires the same degree of freedom (DoF) on each element, so it may lose the $p$-adaptive capability.

In the past two decades, much progress has been made in developing triangular or tetrahedral SEM (TSEM) on unstructured meshes. There are two noticeable trends in designing TSEM. The first is built upon approximation by orthogonal basis related to the collapsed Duffy's transform $[9,10,13,16,24,26]$ and its important variant $[17,18,23]$. The second is based on approximation by nodal basis on special nodal points $[6,11,12,21$, 28]. Here, we elaborate more on the former approach. Firstly, the spectral approximation in triangle using polynomials was much studied (cf. $[3,9,11,19,20,27,28]$ ). Recently, some research efforts have been paid to the non-polynomial spectral approximations in triangle/tetrahedron $[5,16,18,24]$. By using some rectangle-triangle transforms, these spectral methods generate rational or irrational basis functions in triangle from standard tensorial basis functions in rectangle. Two typical rectangle-triangle transforms: Duffy's transform and one-to-one transform (cf. [18]) are frequently adopted. One argument against the Duffy's transform is that the mapped interpolation points are unfavourably clustered near the singular vertex of the triangle. The situation is even severer in the three-dimensional case. To obtain a better distribution of the mapped interpolation points, a new one-to-one transform is designed by pulling one side of the triangle to two sides of the rectangle. As long as the development of the spectral approximations in triangles, more and more attention has been paid on corresponding TSEM. Although the new transform has weaker singularity than the Duffy's transform, it also leads to singular integrand in the calculation of stiffness matrix (cf. $[18,23]$ ). Either a mode basis (cf. $[18,23]$ ) or modified nodal basis [17] is used to handle the singularity. Nevertheless, the special basis functions increase the difficulty in extending to multi-domain cases. Usually, some other techniques (e.g. motar finite element $[2,15]$ ) need to be employed.

This paper is the second of a series on developing TSEM based on the transform [18]. In the first paper [23], a detailed analysis of the logarithmic singularity induced by the transform was conducted and an accurate and stable method to handle such singularities by using mode basis was implemented. Here, we continue to develop a flexible nodal TSEM more applicable to multi-domain cases. The new TSEM is drawn on a mixed formulation using non-polynomial spectral approximations on triangles. Both Duffy's transform and the one-to-one transform can be used to generate non-polynomial basis functions for the method. The main feature of this method is that it is unnecessary to deal with the consistency condition and no singularity will appear in the calculation of the discrete matrices. Actually, the mixed formulation does not involve the stiffness matrix and the singularity in the calculation of other matrices can be eliminated by the Jacobian. Although the mixed formulation introduces a new auxiliary variable, it can be efficiently removed from the discrete linear system due to the fact that the approximated mass matrix is naturally diagonal even in the variable coefficient case. Another main problem to form a spectral element method using non-polynomial spectral approximations in triangles is how to construct and implement a continuous approximation space. We introduce different strategies according to the adopted transform. The proposed TSEM fully enjoys the tensorial structure as QSEM. Hence an efficient implementation can be expected. In theoretical aspect, the $H^{1}$ - 
norm interpolation error estimate presented in this paper contributes to the completion of the approximation theory started in [23]. In addition, an $h p$ error analysis is performed for the proposed TSEM.

The rest of the paper is organized as follows. In Section 2, the rectangle-triangle transforms are introduced to be the extreme cases of the standard transform between quadrilaterals. By using these transforms, we then define the spectral element space on unstructured triangular mesh. Theoretical analysis for interpolations in triangular spectral element space is performed in Section 3. $H^{1}$-norm interpolation error estimate is obtained. In Section 4, we present the numerical formulation of the triangular spectral element method and conduct an $h-p$ error analysis. Various numerical results are given to show the accuracy and flexibility.

\section{Triangular spectral element space}

In this section, we first briefly introduce the rectangle-triangle transform by starting with the typical transform between quadrilaterals. Then we present some details for the construction of conformable spectral element spaces on unstructured triangular mesh by using two typical $\square \rightarrow \triangle$ transforms $T_{0}$ and $T_{1 / 2}$. Implementation techniques for ensuring the conformability in different cases are discussed. The $h-p$ interpolation error estimates in both $L^{2}$ and $H^{1}$ norms will be provided for the general transform $T_{\theta}$ with $0 \leq \theta \leq 1$ at the end of this section.

\subsection{The rectangle-triangle transform}

Throughout this paper, we denote by

$$
\triangle:=\{(\widehat{x}, \widehat{y}): 0<\widehat{x}, \widehat{y}, \widehat{x}+\widehat{y}<1\} \subset \mathbb{R}^{2}, \quad \square:=\{(\xi, \eta):-1<\xi, \eta<1\} \subset \mathbb{R}^{2},
$$

the reference triangle and the reference square, respectively. Here, $\mathbb{R}$ is the set of all real numbers. The vertices $\widehat{P}_{1}(-1,-1), \widehat{P}_{2}(1,-1), \widehat{P}_{3}(1,1)$ and $\widehat{P}_{4}(-1,1)$ of the square $\square$ are denoted simply by $\left\{\widehat{P}_{i}\left(\xi_{i}, \eta_{i}\right)\right\}_{i=1}^{4}$. Given an arbitrary convex quadrilateral $\diamond$ with vertices denoted by $\left\{P_{i}\left(x_{i}, y_{i}\right)\right\}_{i=1}^{4}$, we define the constants:

$$
\begin{aligned}
& \alpha_{1}=\frac{1}{4} \sum_{i=1}^{4} x_{i} \xi_{i} \eta_{i}, \quad \alpha_{2}=\frac{1}{4} \sum_{i=1}^{4} x_{i} \xi_{i}, \quad \alpha_{3}=\frac{1}{4} \sum_{i=1}^{4} x_{i} \eta_{i}, \quad \alpha_{4}=\frac{1}{4} \sum_{i=1}^{4} x_{i}, \\
& \beta_{1}=\frac{1}{4} \sum_{i=1}^{4} y_{i} \xi_{i} \eta_{i}, \quad \beta_{2}=\frac{1}{4} \sum_{i=1}^{4} y_{i} \xi_{i}, \quad \beta_{3}=\frac{1}{4} \sum_{i=1}^{4} y_{i} \eta_{i}, \quad \beta_{4}=\frac{1}{4} \sum_{i=1}^{4} y_{i} .
\end{aligned}
$$

It is well known that the iso-parametric transform:

$$
F: x=\alpha_{1} \xi \eta+\alpha_{2} \xi+\alpha_{3} \eta+\alpha_{4}, \quad y=\beta_{1} \xi \eta+\beta_{2} \xi+\beta_{3} \eta+\beta_{4}, \quad \forall(\xi, \eta) \in \square
$$

is an one-to-one transform from $\square$ to $\diamond$. Its Jacobian is

$$
J=|\mathbb{J}|=\operatorname{det}\left(\frac{\partial(x, y)}{\partial(\xi, \eta)}\right)=\left|\begin{array}{ll}
\alpha_{1} \eta+\alpha_{2} & \beta_{1} \eta+\beta_{2} \\
\alpha_{1} \xi+\alpha_{3} & \beta_{1} \xi+\beta_{3}
\end{array}\right|=D_{1} \xi+D_{2} \eta+D_{3},
$$




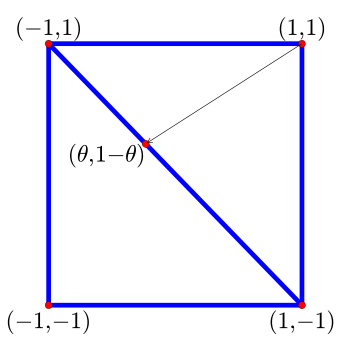

(a) $T_{\theta}: \square \mapsto \triangle$

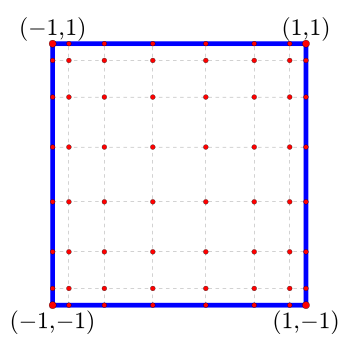

(b) LGL points on $\square$

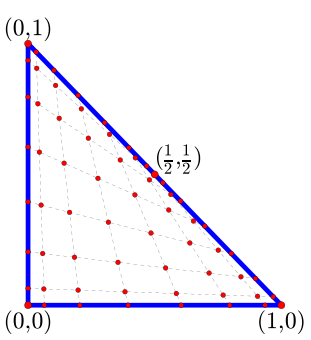

(c) LGL points by $T_{1 / 2}$

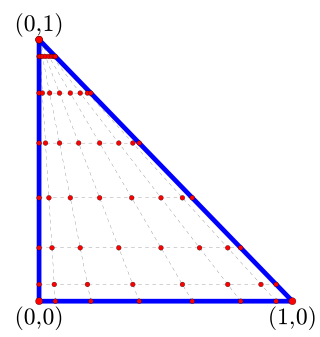

(d) LGL points by $T_{0}$

Figure 1: The rectangle-quadrilateral transforms.

where

$$
D_{1}=\left|\begin{array}{ll}
\alpha_{2} & \beta_{2} \\
\alpha_{1} & \beta_{1}
\end{array}\right|, \quad D_{2}=\left|\begin{array}{ll}
\alpha_{1} & \beta_{1} \\
\alpha_{3} & \beta_{3}
\end{array}\right|, \quad D_{3}=\left|\begin{array}{ll}
\alpha_{2} & \beta_{2} \\
\alpha_{3} & \beta_{3}
\end{array}\right| .
$$

If all inner angles of the quadrilateral $\diamond$ is less than $\pi$, we have $J>0$ and the inverse of the Jacobian matrix $\mathbb{J}$ is

$$
\mathbb{J}^{-1}=\frac{1}{J}\left[\begin{array}{cc}
\beta_{1} \xi+\beta_{3} & -\beta_{1} \eta-\beta_{2} \\
-\alpha_{1} \xi-\alpha_{3} & \alpha_{1} \eta+\alpha_{2}
\end{array}\right] .
$$

Choosing $P_{1}(0,0), P_{2}(1,0), P_{3}(\theta, 1-\theta), P_{4}(0,1)$ in the transform defined in (2.1)-(2.2), we have

$$
\alpha_{1}=\alpha_{3}=\frac{\theta-1}{4}, \quad \alpha_{2}=\alpha_{4}=\frac{\theta+1}{4}, \quad \beta_{1}=\beta_{2}=-\frac{\theta}{4}, \quad \beta_{3}=\beta_{4}=\frac{2-\theta}{4},
$$

which leads to $\square \mapsto \triangle$ transform $T_{\theta}(0 \leq \theta \leq 1)$ (cf. [23]):

$$
\widehat{x}=\frac{1+\xi}{2} \frac{2-(1-\theta)(1+\eta)}{2}, \quad \widehat{y}=\frac{1+\eta}{2} \frac{2-\theta(1+\xi)}{2}, \quad \forall(\xi, \eta) \in \square .
$$

Here, the reference triangle $\triangle$ is seen as a quadrilateral with inner angle equal to $\pi$. We see that this transform pulls the hypotenuse of $\triangle$ into two edges of $\square$ at the point $(\theta, 1-\theta)$. Substituting (2.6) into (2.4) and then using formula (2.3) gives the Jacobian of $T_{\theta}$ :

$$
J_{\theta}(\xi, \eta)=\operatorname{det}\left(\mathbb{J}_{\theta}\right)=\left|\begin{array}{ll}
\frac{(\theta-1) \eta+\theta+1}{4} & \frac{-\theta(\eta+1)}{4} \\
\frac{(\theta-1)(\xi+1)}{4} & \frac{-\theta \xi+2-\theta}{4}
\end{array}\right|=\frac{1-(\theta \xi+(1-\theta) \eta)}{8} .
$$

Further, the Jacobian in $(\widehat{x}, \widehat{y})$ coordinates can be derived from expression (2.7), i.e.,

$$
J_{\theta}(\widehat{x}, \widehat{y})=\frac{\sqrt{(\theta \widehat{x}-(1-\theta) \widehat{y})^{2}+1-2 \theta \widehat{x}-2(1-\theta) \widehat{y}}}{4} .
$$


Clearly, the Jacobian (2.8) has zeros in the reference square $\square$ and the inverse of $\mathbb{J}_{\theta}$ :

$$
\mathbb{J}_{\theta}^{-1}=\left[\begin{array}{ll}
\frac{\partial \xi}{\partial \widehat{x}} & \frac{\partial \eta}{\partial \widehat{x}} \\
\frac{\partial \xi}{\partial \widehat{y}} & \frac{\partial \eta}{\partial \widehat{y}}
\end{array}\right]=\frac{1}{4 J_{\theta}}\left[\begin{array}{cc}
-\theta \xi+2-\theta & \theta(\eta+1) \\
-(\theta-1)(\xi+1) & (\theta-1) \eta+\theta+1
\end{array}\right]
$$

is not always well defined in the reference square.

The Duffy's transform (cf. [10]) $T_{0}$ :

$$
\widehat{x}=\frac{1}{4}(1+\xi)(1-\eta), \quad \widehat{y}=\frac{1}{2}(1+\eta), \quad \forall(\xi, \eta) \in \square
$$

is the limitting case of (2.7) with $\theta=0$. The new $\square \rightarrow \triangle$ transform $T_{\frac{1}{2}}$ :

$$
\widehat{x}=\frac{1}{8}(1+\xi)(3-\eta), \quad \widehat{y}=\frac{1}{8}(3-\xi)(1+\eta), \quad \forall(\xi, \eta) \in \square
$$

introduced in [18] is another special case of (2.7) with $\theta=\frac{1}{2}$. Their Jacobian matrices are given by

$$
J_{0}=\left|\begin{array}{cc}
\frac{1-\eta}{4} & 0 \\
-\frac{\xi+1}{4} & \frac{1}{2}
\end{array}\right|=\frac{1-\eta}{8}, \quad J_{\frac{1}{2}}=\left|\begin{array}{cc}
\frac{3-\eta}{8} & -\frac{\eta+1}{8} \\
-\frac{\xi+1}{8} & \frac{3-\xi}{8}
\end{array}\right|=\frac{2-\xi-\eta}{16}
$$

and their inverses are

$$
\mathbb{J}_{0}^{-1}=\frac{2}{1-\eta}\left[\begin{array}{cc}
2 & 0 \\
\xi+1 & 1-\eta
\end{array}\right], \quad \mathbb{J}_{\frac{1}{2}}^{-1}=\frac{2}{2-\xi-\eta}\left[\begin{array}{cc}
3-\xi & \eta+1 \\
\xi+1 & 3-\eta
\end{array}\right] .
$$

For $0<\theta<1$, we have the inverse transform $T_{\theta}^{-1}$ :

$$
\begin{aligned}
\xi & =\frac{1-\theta+(\theta \widehat{x}-(1-\theta) \widehat{y})-4 J_{\theta}(\widehat{x}, \widehat{y})}{\theta}, \\
\eta & =\frac{\theta-(\theta \widehat{x}-(1-\theta) \widehat{y})-4 J_{\theta}(\widehat{x}, \widehat{y})}{1-\theta},
\end{aligned}
$$

where $J_{\theta}(\widehat{x}, \widehat{y})$ is defined in (2.9). On the other hand, we have inverse transforms:

$$
T_{0}^{-1}:\left\{\begin{array}{l}
\xi=\frac{2 \widehat{x}}{1-\widehat{y}}-1, \\
\eta=2 \widehat{y}-1,
\end{array} \quad T_{1}^{-1}:\left\{\begin{array}{l}
\xi=2 \widehat{x}-1, \\
\eta=\frac{2 \widehat{y}}{1-\widehat{x}}-1
\end{array}\right.\right.
$$

for limiting cases $\theta=0,1$, respectively. 


\subsection{Construction of triangular spectral element space}

Hereafter, let $I=(-1,1)$, and for any integer $p \geq 1$, denote by $P_{p}(I)$ the set of all algebraic polynomials of degree at most $p$. Two standard polynomial spaces on the reference square $\square$ and $\triangle$ are

$$
\mathscr{P}_{p}(\triangle):=\operatorname{span}\left\{\widehat{x}^{i} \widehat{y}^{j}: 0 \leq i+j \leq p\right\}, \quad \mathscr{Q}_{p}(\square):=\left(P_{p}(I)\right)^{2} .
$$

The following property (cf. [23]) shows that all polynomials on $\triangle$ are still polynomials on the reference $\square$.

Proposition 2.1. Let $T_{\theta}$ be the rectangle-triangle transform defined in (2.7), and $\mathscr{P}_{k}(\triangle) \circ$ $T_{\theta}=\left\{P \circ T_{\theta}: P \in \mathscr{P}_{k}(\triangle)\right\}$. We have $\mathscr{P}_{p}(\triangle) \circ T_{\theta} \subset \mathscr{Q}_{p}(\square)$ for $0 \leq \theta \leq 1$.

Define the space

$$
Y_{p, \theta}(\triangle)=\mathscr{Q}_{p}(\square) \circ T_{\theta}^{-1}=\left(P_{p}(I)\right)^{2} \circ T_{\theta}^{-1},
$$

which consists of the images of the tensorior polynomials on $\square$ under the transform $T_{\theta}^{-1}$. As a direct consequence of Proposition 2.1,

$$
\mathscr{P}_{p}(\triangle) \subseteq Y_{p, \theta}(\triangle) \quad \text { forall } \theta \in[0,1] .
$$

The inverse transforms (2.15) and (2.16) imply that $Y_{p, \theta}(\triangle)$ contains not only polynomials, but also some special irrational functions. According to the definition (2.18), the basis functions of $Y_{p, \theta}(\triangle)$ can be obtained by applying the transform $T_{\theta}^{-1}$ to the basis functions of $\mathscr{Q}_{p}(\square)$. In practice, we use the nodal basis of $\mathscr{Q}_{p}(\square)$. Denote by $\left\{\zeta_{i}\right\}_{i=0}^{p}$ the LegendreGauss-Lobatto (LGL) points in $I$ and $\left\{h_{m}(\zeta)\right\}_{m=0}^{p}$ the corresponding Lagrange interpolating basis polynomials, i.e., $h_{m} \in P_{p}(I)$ and $h_{m}\left(\zeta_{n}\right)=\delta_{m n}$ (where $\delta_{m n}$ is the Kronecker Delta symbol). Then

$$
\mathscr{Q}_{p}(\square)=\operatorname{span}\left\{\varphi_{m n}: \varphi_{m n}(\xi, \eta)=h_{m}(\xi) h_{n}(\eta), 0 \leq m, n \leq p\right\} .
$$

This gives the nodal basis of $Y_{p, \theta}(\triangle)$ :

$$
Y_{p, \theta}(\triangle)=\operatorname{span}\left\{\psi_{m n}: \psi_{m n}(\widehat{x}, \widehat{y})=\phi_{m n} \circ T_{\theta}^{-1}(\widehat{x}, \widehat{y}), \quad 0 \leq m, n \leq p\right\} .
$$

Let $\mathscr{T}_{h}:=\{K\}$ denote a shape regular quasi-uniform triangular mesh of a polygonal domain $\Omega$. Denote by $h_{K}=\operatorname{diam}(K)$ the diameter of element $K, h=\max \left\{h_{K}\right\} . \mathscr{T}_{h}$ is a shape regular quasi-uniform mesh if there exists positive constants $c_{0}$ and $c_{1}$ such that

$$
\max _{K \in \mathscr{T}_{h}} \frac{h_{K}^{2}}{|K|} \leq c_{0}, \quad \frac{h}{\min _{K \in \mathscr{T}_{h}} h_{K}} \leq c_{1},
$$

where $|K|$ is the measure of $K$. The triangular spectral element space on $\mathscr{T}_{h}$ is defined as

$$
S_{h, p}^{\theta}\left(\mathscr{T}_{h}\right)=\left\{v_{h} \in H^{1}(\Omega):\left.v_{h}\right|_{K} \circ F_{K} \in Y_{p, \theta}(\triangle), \forall K \in \mathscr{T}_{h}\right\},
$$




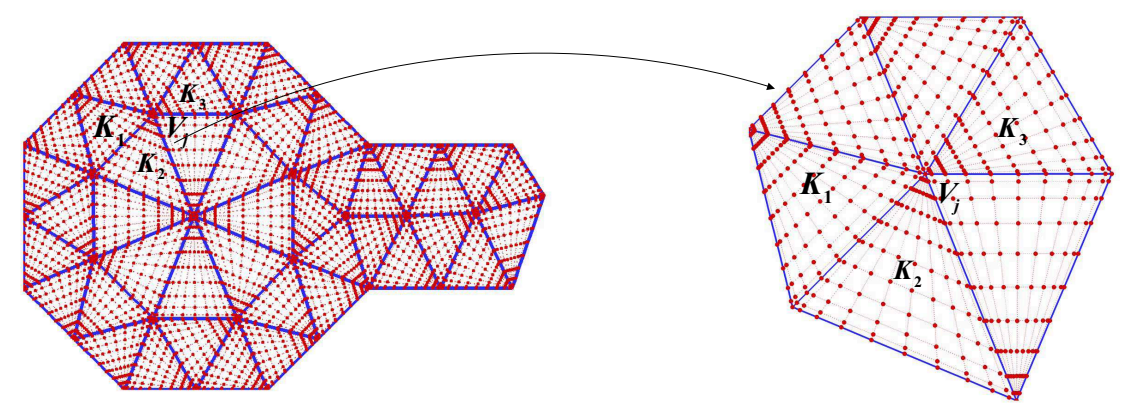

Figure 2: Physical node distribution via $T_{0}$ in an unstructured mesh $(p=10)$.

where $F_{K}: \triangle \rightarrow K$ is the standard affine mapping from the reference triangle $\Delta$ to a physical element $K \in \mathscr{T}_{h}$. Although the definition of triangular spectral element space $S_{h, p}^{\theta}\left(\mathscr{T}_{h}\right)$ follows the classic way, some cases have to be taken to impose the continuity across elements due to the singularity introduced by the transform $T_{\theta}$. In what follows, we elaborate on two typical cases using transforms $T_{0}, T_{1 / 2}$ and show how to construct continuous global basis functions from local basis functions on arbitrary unstructured mesh $\mathscr{T}_{h}$.

Case I: The Duffy's transform $T_{0}$. Due to the collapsed Duffy's transform $T_{0}$, the nodes $\left\{\left(\widehat{x}_{m}, \widehat{y}_{n}\right)\right\}_{m, n=0}^{p}$ in the reference triangle $\triangle$ clustered near the singular vertex $(0,1)$ and all nodes $\left\{\left(\widehat{x}_{m}, \widehat{y}_{n}\right)\right\}_{m, n=0}^{p}$ collapsed to one node at $(0,1)$. After applying the standard affine mapping $F_{K}$, the corresponding nodes $\left\{\left(x_{m}^{K}, x_{n}^{K}\right)\right\}_{m, n=0}^{p}$ in the physical element $K$ also clustered near one vertex of $K$. The physical nodes $\left\{\left(x_{m}^{K}, x_{n}^{K}\right)\right\}_{m, n=0}^{p}$ may be clustered near any vertex of the physical element $K$ due to the rotational free of the mapping $F_{K}$. In an unstructured mesh a vertex $V_{j}$ may be shared by several elements see Fig. 2 . In the attached elements, some elements have nodes clustered near $V_{j}$ and the others do not, e.g., $K_{2}$ and $K_{3}$ in Fig. 2 have nodes clustered near $V_{j}$. Although there are nodes clustered near vertices, the nodes from neighboring elements matched with each other on their common edge, e.g., in Fig. 2 nodes in $K_{1}$ and $K_{2}$ mathed on their common edge. For ensuring the continuity of the numerical solution, we only need to handle the nodes clustered near vertices. We merge all nodes clustered at a given vertex $V_{j}$ to one node. This can be done by assigning one global index to all nodes corresponding to $V_{j}$ in the implementation. For example, there are $4+2(p+1)$ nodes share one global index determined by the vertex $V_{j}$ in Fig. 2. It is worthy pointing out that this implementation technique is equivalent to using polynomial space (cf. [24])

$$
\widetilde{Q}_{p}(\square)=\left\{\phi \in P_{p}(I) \times P_{p}(I): \partial_{\xi} \phi(\xi, 1)=0\right\} .
$$

The basis function $h_{p}(\eta) \circ T_{0}^{-1}(\widehat{x}, \widehat{y})$ is acutally used for node at vertex $(0,1)$ in the reference triangle.

Case II: The one-to-one transform $T_{1 / 2}$. The transform $T_{1 / 2}$ maps the hypotenuse 


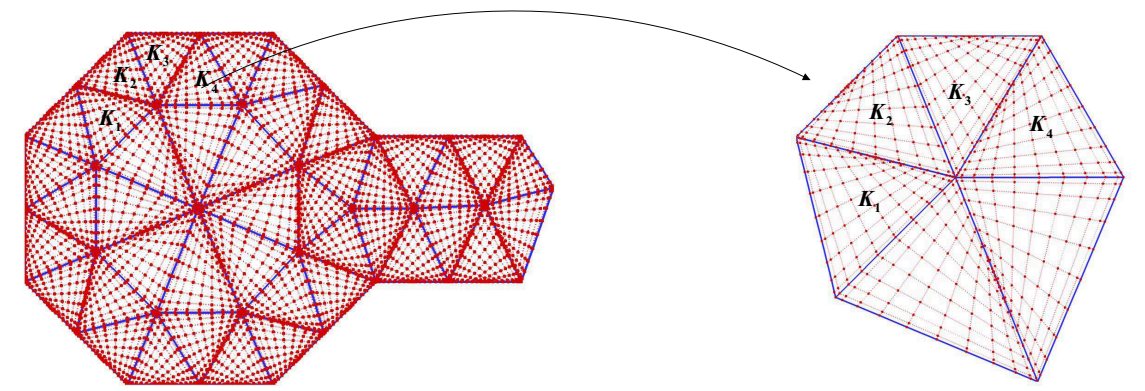

Figure 3: Inconsistent physical node distribution via $T_{1 / 2}$ and arbitrary elemental affine mappings ( $p=$ 10).

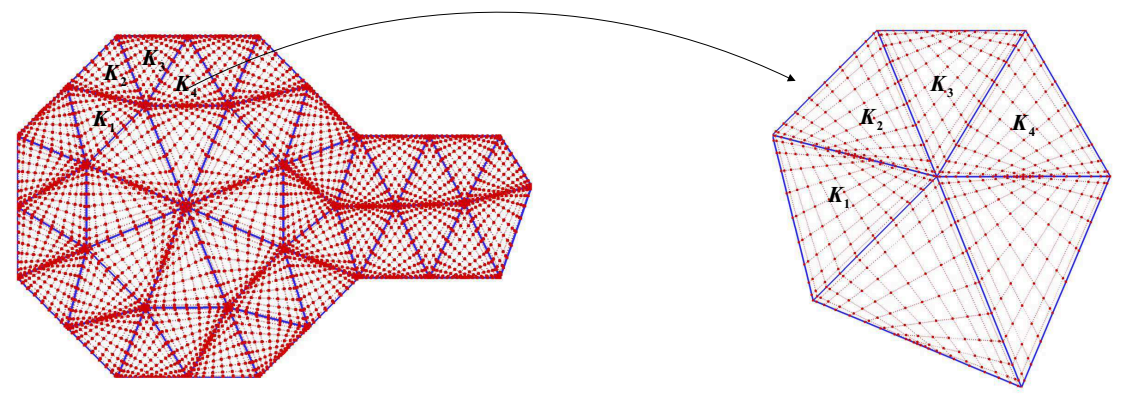

Figure 4: Consistent physical node distribution via $T_{1 / 2}$ and adjusted elemental affine mappings $(p=10)$.

of the reference triangle $\Delta$ to two sides of the reference rectangle $\square$. Therefore, the number of nodes on the hypotenuse of $\triangle$ is twice as many as that on other two sides due to the transform $T_{1 / 2}$, see Fig. 1 (c). As a result, each physical element $K$ inherits such inconsistency. Since affine mappings $F_{K}$ randomly map edges in $K$ to hypotenuse and other two edges of refrence triangle $\triangle$, the images of a common edge of two neighboring elements may be different edges in $\triangle$. If one maps the common edge to the hypotenuse of $\triangle$ and the other maps it to another different side of $\triangle$, then the common edge have differnet number of nodes from two sides (see $K_{3}$ and $K_{4}$ in Fig. 3). This inconsistency will cause great difficulty in the contruction of continuous spectral element space $S_{h, p}^{\theta}\left(\mathscr{T}_{h}\right)$. Fortunately, the inconsistency can be avoid by configuring the elemental affine mappings $F_{K}$ properly. For any interior edge $e_{i j}=K_{i} \cap K_{j}$, we choose elemental mappings $F_{K_{i}}$ and $F_{K_{j}}$ such that $F_{K_{j}}$ mapps $e_{i j}$ to the hypotenuse of $\triangle$ if and only if $F_{K_{i}}$ maps $e_{i j}$ to the hypotenuse of $\triangle$, see Fig. 4. The required elemental mapping configuration can be determined by the mesh beforehand. Numerical examples show that configurations without producing node inconsistency are available even for complicate unstructured meshes (see, examples given in Subsection 4.3). Once we have the node consistent elemental mappings ready, the implementation of TSEM using transform $T_{1 / 2}$ becomes quite simple. We just view a given triangular mesh as a deformed quadrilateral mesh by view each triangular element as a deformed quadrilateral element in which the middle point of the hypotenuse becomes a new vertex. 


\section{The approximation properties of $S_{h, p}^{\theta}\left(\mathscr{T}_{h}\right)$}

We first introduce some notions. The weighted Sobolev space $H_{w}^{r}(\Omega)$ with $r>0$ is defined as in Adams [1], and its norm and semi-norm are denoted by $\|\cdot\|_{r, w, \Omega}$ and $|\cdot|_{r, w, \Omega}$, respectively. In particular, if $r=0$, we denote the inner product and norm of $L_{w}^{2}(\Omega)$ by $(\cdot, \cdot)_{w, \Omega}$ and $\|\cdot\|_{w, \Omega}$, respectively. Moreover, if $w \equiv 1$, we drop it from the notion.

With the LGL points $\left\{\zeta_{j}\right\}_{j=0}^{p}$ and corresponding Lagrangian polynomial basis $\left\{h_{j}(\zeta)\right\}_{j=0}^{p}$, the LGL interpolation of given function $v \in C(\bar{I})$ is defined as

$$
\left(I_{p}^{\zeta} v\right)(\zeta)=\sum_{j=0}^{p} v\left(\zeta_{j}\right) h_{j}(\zeta) \in P_{p}, \quad \forall \zeta \in \bar{I} .
$$

Moreover, the following estimates hold.

Lemma 3.1. If $v \in H^{r}(I)$ with $r \geq 1$ and $\mu=0,1$, we have

$$
\left\|\left(I_{p}^{\zeta} v-v\right)^{(\mu)}\right\|_{L^{2}(I)} \leq C p^{\mu-r}\left\|\left(1-\zeta^{2}\right)^{(r-1) / 2} v^{(r)}\right\|_{L^{2}(I)} .
$$

Proof. For $\mu=0$, it is the direct result of Theorem 3.44 in [25]. To prove the estimate for $\mu=1$, one verifies that

$$
v^{\prime} \in B_{0,0}^{r-1}(I):=\left\{w \in L^{2}(I): w^{(k)} \in L_{\omega^{k, k}}^{2}(I), 0 \leq k \leq r-1\right\}
$$

for any $v \in H^{r}(I), r \geq 1$. Here

$$
\omega^{k, k}(\zeta)=(1-\zeta)^{k}(1+\zeta)^{k}
$$

is the Jacobi weight function. Applying the estimate in Lemma 2.3 in [24], we obtain the inequality for $\mu=1$.

For any $\widehat{u} \in C(\triangle)$, define interpolation $\mathbb{I}_{p, \theta} \widehat{u}(\widehat{x}, \widehat{y}) \in Y_{p, \theta}(\triangle)$ such that

$$
\mathbb{I}_{p, \theta} \widehat{u}\left(\widehat{x}_{i}, \widehat{y}_{j}\right)=\widehat{u}\left(\widehat{x}_{i}, \widehat{y}_{j}\right), \quad 0 \leq i, j \leq p,
$$

where $\left\{\left(\widehat{x}_{i}, \widehat{y}_{j}\right)=T_{\theta}\left(\xi_{i}, \eta_{j}\right)\right\}$ are the mapped LGL points. Let

$$
\widetilde{u}(\xi, \eta)=\left(\widehat{u} \circ T_{\theta}\right)(\xi, \eta) .
$$

One verifies readily that

$$
\left(I_{p, \theta} \widehat{u}\right)(\widehat{x}, \widehat{y})=\left(\left(I_{p}^{\xi} I_{p}^{\eta} \widetilde{u}\right) \circ T_{\theta}^{-1}\right)(\widehat{x}, \widehat{y})=\left(\left(I_{p}^{\eta} I_{p}^{\xi} \widetilde{u}\right) \circ T_{\theta}^{-1}\right)(\widehat{x}, \widehat{y}) .
$$

It is worthy of pointing out that an interpolation with LGL in $\xi$-direction and JGR points in $\eta$-direction for the case $\theta=0$ was investigated in [24]. Nevertheless, it cannot be used to define an interpolation in the conformal spectral element space $S_{h, p}^{0}\left(\mathscr{T}_{h}\right)$ due to the usage of JGR points in $\eta$-direction. Here the interpolation $\mathbb{I}_{p, \theta}$ uses the LGL points in both $\xi$ and 
$\eta$-directions. Once proper affine mappings $F_{K}$ is set for $0<\theta<1$ as stated in the last section, the global interpolation of any given function $u \in C(\bar{\Omega})$ in the space $S_{h, p}^{\theta}\left(\mathscr{T}_{h}\right)$ can be directly defined as

$$
\left(\mathscr{I}_{h, p}^{\theta} u\right)(x, y)=\left(\mathbb{I}_{p, \theta} \widehat{u}\right)\left(F_{K}^{-1}(x, y)\right), \quad(x, y) \in K, \quad \forall K \in \mathscr{T}_{h},
$$

where

$$
\widehat{u}\left(F_{K}^{-1}(x, y)\right)=u(x, y), \quad \forall(x, y) \in K, \quad K \in \mathscr{T}_{h} .
$$

The continuity of $\mathscr{I}_{h, p}^{\theta} u$ in $\Omega$ can be verified for all $0 \leq \theta \leq 1$.

An $L^{2}$-error estimate for $\mathbb{I}_{p, \frac{1}{2}}$ has been proved in [23]. However, an $H^{1}$-error estimate is usually indispensable in the error analysis of the spectral element method. By using Lemma 3.1, we can derive the following $H^{1}$-error estimate.

Theorem 3.1. Suppose $\widehat{u} \in H^{2}(\triangle)$. Then

$$
\left\|I_{p, \theta} \widehat{u}-\widehat{u}\right\|_{\mu, \Delta} \leq C p^{\mu-2}\left(|\widehat{u}|_{2, \Delta}+\left\|\left(\partial_{\widehat{y}}-\partial_{\widehat{x}}\right)^{2} \widehat{u}\right\|_{J_{\theta}^{-1}, \Delta}+\|\widehat{\nabla} \widehat{u}\|_{J_{\theta}^{-1}, \Delta}\right), \quad \mu=0,1
$$

for $0<\theta<1$, where $J_{\theta}$ is the Jacobian as defined in (2.8).

Moreover, if $\widehat{u} \in H^{r}(\triangle)$ with $r \geq 3$, then

$$
\left\|I_{p, \theta} \widehat{u}-\widehat{u}\right\|_{\mu, \Delta} \leq C p^{\mu-r}\left(|\widehat{u}|_{r, \Delta}+|\widehat{u}|_{r-1, \Delta}\right), \quad \mu=0,1
$$

for $0 \leq \theta \leq 1$.

Proof. Let $I_{d}$ be the identity operator and

$$
\widetilde{u}(\xi, \eta)=\left(\widehat{u} \circ T_{\theta}\right)(\xi, \eta) .
$$

From (2.8) and (2.10), we have

$$
J_{\theta}, J_{\theta} \frac{\partial \xi}{\partial \widehat{x}}, J_{\theta} \frac{\partial \xi}{\partial \widehat{y}}, J_{\theta} \frac{\partial \eta}{\partial \widehat{x}}, J_{\theta} \frac{\partial \eta}{\partial \widehat{y}}
$$

uniformly bounded in $\square$ for all $0 \leq \theta \leq 1$. Then together with (3.4), we derive that

$$
\begin{aligned}
& \quad\left\|I_{p, \theta} \widehat{u}-\widehat{u}\right\|_{\triangle}^{2}=\left\|\left(I_{p}^{\xi} I_{p}^{\eta} \widetilde{u}-\widetilde{u}\right) J_{\theta}\right\|_{\square}^{2} \leq C\left\|I_{p}^{\xi} I_{p}^{\eta} \widetilde{u}-\widetilde{u}\right\|_{\square}^{2}, \\
& \left\|I_{p, \theta} \widehat{u}-\widehat{u}\right\|_{\triangle}^{2}+\left\|\partial_{\widehat{x}}\left(I_{p, \theta} \widehat{u}-\widehat{u}\right)\right\|_{\triangle}^{2}+\left\|\partial_{\widehat{y}}\left(I_{p, \theta} \widehat{u}-\widehat{u}\right)\right\|_{\triangle}^{2} \\
& =\left\|\left(I_{p}^{\xi} I_{p}^{\eta} \widetilde{u}-\widetilde{u}\right) J_{\theta}\right\|_{\square}^{2}+\left\|\partial_{\xi}\left(I_{p}^{\xi} I_{p}^{\eta} \widetilde{u}-\widetilde{u}\right) \frac{\partial \xi}{\partial \widehat{x}} J_{\theta}+\partial_{\eta}\left(I_{p}^{\xi} I_{p}^{\eta} \widetilde{u}-\widetilde{u}\right) \frac{\partial \eta}{\partial \widehat{x}} J_{\theta}\right\|_{\square}^{2} \\
& \quad+\left\|\partial_{\xi}\left(I_{p}^{\xi} I_{p}^{\eta} \widetilde{u}-\widetilde{u}\right) \frac{\partial \xi}{\partial \widehat{y}} J_{\theta}+\partial_{\eta}\left(I_{p}^{\xi} I_{p}^{\eta} \widetilde{u}-\widetilde{u}\right) \frac{\partial \eta}{\partial \widehat{y}} J_{\theta}\right\|_{\square}^{2} \\
& \leq C\left(\left\|I_{p}^{\xi} I_{p}^{\eta} \widetilde{u}-\widetilde{u}\right\|_{\square}^{2}+\left\|\partial_{\xi}\left(I_{p}^{\xi} I_{p}^{\eta} \widetilde{u}-\widetilde{u}\right)\right\|_{\square}^{2}+\left\|\partial_{\eta}\left(I_{p}^{\xi} I_{p}^{\eta} \widetilde{u}-\widetilde{u}\right)\right\|_{\square}^{2}\right) .
\end{aligned}
$$


Using the decomposition

$$
I_{p}^{\xi} I_{p}^{\eta} \widetilde{u}-\widetilde{u}=\left(I_{p}^{\xi}-I_{d}\right)\left(I_{p}^{\eta}-I_{d}\right) \widetilde{u}+\left(I_{p}^{\xi}-I_{d}\right) \widetilde{u}+\left(I_{p}^{\eta}-I_{d}\right) \widetilde{u},
$$

and the approximation results in Lemma 3.1, we have estimates:

$$
\begin{aligned}
& \left\|I_{p}^{\xi} I_{p}^{\eta} \widetilde{u}-\widetilde{u}\right\|_{\triangle} \leq C\left(\left\|\left(I_{p}^{\xi}-I_{d}\right)\left(I_{p}^{\eta}-I_{d}\right) \widetilde{u}\right\|_{\square}+\left\|\left(I_{p}^{\xi}-I_{d}\right) \widetilde{u}\right\|_{\square}+\left\|\left(I_{p}^{\eta}-I_{d}\right) \widetilde{u}\right\|_{\square}\right) \\
\leq & C p^{-1}\left\|\left(I_{p}^{\xi}-I_{d}\right) \partial_{\eta} \widetilde{u}\right\|_{\square}+C\left(\left\|\left(I_{p}^{\xi}-I_{d}\right) \widetilde{u}\right\|_{\square}+\left\|\left(I_{p}^{\eta}-I_{d}\right) \widetilde{u}\right\|_{\square}\right) \\
\leq & C p^{-r}\left(\left\|\left(1-\xi^{2}\right)^{\frac{(r-2)}{2}} \partial_{\eta} \partial_{\xi}^{r-1} \widetilde{u}\right\|_{\square}+\left\|\left(1-\xi^{2}\right)^{\frac{(r-1)}{2}} \partial_{\xi}^{r} \widetilde{u}\right\|_{\square}+\left\|\left(1-\eta^{2}\right)^{\frac{(r-1)}{2}} \partial_{\eta}^{r} \widetilde{u}\right\|_{\square}\right), \quad(3.9 \\
& \left\|\partial_{\xi}\left(I_{p}^{\xi} I_{p}^{\eta} \widetilde{u}-\widetilde{u}\right)\right\|_{\square} \leq C\left\|\partial_{\xi}\left[\left(I_{p}^{\xi}-I_{d}\right)\left(I_{p}^{\eta}-I_{d}\right) \widetilde{u}\right]+\partial_{\xi}\left[\left(I_{p}^{\xi}-I_{d}\right) \widetilde{u}\right]+\left(I_{p}^{\eta}-I_{d}\right) \partial_{\xi} \widetilde{u}\right\|_{\square} \\
\leq & C\left(\left\|\partial_{\xi}\left[\left(I_{p}^{\xi}-I_{d}\right)\left(I_{p}^{\eta}-I_{d}\right) \widetilde{u}\right]\right\|_{\square}+\left\|\partial_{\xi}\left[\left(I_{p}^{\xi}-I_{d}\right) \widetilde{u}\right]\right\|_{\square}+\left\|\left(I_{p}^{\eta}-I_{d}\right) \partial_{\xi} \widetilde{u}\right\|_{\square}\right) \\
\leq & C\left\|\left(I_{p}^{\eta}-I_{d}\right) \partial_{\xi} \widetilde{u}\right\|_{\square}+C p^{1-r}\left\|\left(1-\xi^{2}\right)^{\frac{(r-1)}{2}} \partial_{\xi}^{r} \widetilde{u}\right\|_{\square} \\
\leq & C p^{1-r}\left(\left\|\left(1-\eta^{2}\right)^{\frac{(r-2)}{2}} \partial_{\xi} \partial_{\eta}^{r-1} \widetilde{u}\right\|_{\square}+\left\|\left(1-\xi^{2}\right)^{\frac{(r-1)}{2}} \partial_{\xi}^{r} \widetilde{u}\right\|_{\square}\right), \\
& \left\|\partial_{\eta}\left(I_{p}^{\xi} I_{p}^{\eta} \widetilde{u}-\widetilde{u}\right)\right\|_{\square} \leq C p^{1-r}\left(\left\|\left(1-\xi^{2}\right)^{\frac{(r-2)}{2}} \partial_{\eta} \partial_{\xi}^{r-1} \widetilde{u}\right\|_{\square}+\left\|\left(1-\eta^{2}\right)^{\frac{(r-1)}{2}} \partial_{\eta}^{r} \widetilde{u}\right\|_{\square}\right) .
\end{aligned}
$$

Next, we bound the four terms

$$
\begin{array}{ll}
E_{r, \square}^{(1)}(\widetilde{u})=\left\|\left(1-\xi^{2}\right)^{(r-1) / 2} \partial_{\xi}^{r} \widetilde{u}\right\|_{\square}, & E_{r, \square}^{(2)}(\widetilde{u})=\left\|\left(1-\eta^{2}\right)^{(r-1) / 2} \partial_{\eta}^{r} \widetilde{u}\right\|_{\square} \\
E_{r, \square}^{(3)}(\widetilde{u})=\left\|\left(1-\xi^{2}\right)^{(r-2) / 2} \partial_{\eta} \partial_{\xi}^{r-1} \widetilde{u}\right\|_{\square}, & E_{r, \square}^{(4)}(\widetilde{u})=\left\|\left(1-\eta^{2}\right)^{(r-2) / 2} \partial_{\xi} \partial_{\eta}^{r-1} \widetilde{u}\right\|_{\square}
\end{array}
$$

in the right-hand sides using norm of $u$ on $\triangle$ one by one. In the following estimate, we shall only consider the case $\theta=0$. Other cases $\theta \neq 0$ can be estimated in similar way (ref. [23]).

Recalling (2.8), we obtain from a direct calculation that

$$
\begin{array}{ll}
\partial_{\xi} \widehat{x}=\frac{1-\eta}{4}, & \partial_{\xi} \widehat{y}=0, \\
\partial_{\eta} \widehat{x}=-\frac{1+\xi}{4}, & \partial_{\eta} \widehat{y}=\frac{1}{2}, \\
\partial_{\xi} \tilde{u}=\left(\frac{1-\eta}{4}\right) \partial_{\widehat{x}} \widehat{u}, & \frac{\partial \widetilde{u}}{\partial \eta}=-\frac{1+\xi}{4} \partial_{\widehat{x}} \widehat{u}+\frac{1}{2} \partial_{\widehat{y}} \widehat{u} .
\end{array}
$$

Define

$$
a(\eta, k):=\left(\frac{1-\eta}{4}\right)^{k}, \quad b(\xi, k)=\left(-\frac{1+\xi}{4}\right)^{k}
$$


Then we have

$$
\begin{gathered}
\partial_{\xi}^{r} \widetilde{u}=a(\eta, r) \partial_{\widehat{x}}^{r} \widehat{u}, \quad \partial_{\eta}^{r} \widetilde{u}=\sum_{k=0}^{r}\left(\begin{array}{l}
r \\
k
\end{array}\right) b(\xi, k)\left(\frac{1}{2}\right)^{r-k} \partial_{\widehat{x}}^{k} \partial_{\widehat{y}}^{r-k} \widehat{u}, \\
\partial_{\xi}^{r-1} \partial_{\eta} \widetilde{u}=\partial_{\eta}\left[a(\eta, r-1) \partial_{\widehat{x}}^{r-1} \widehat{u}\right]=-\frac{r-1}{4} a(\eta, r-2) \partial_{\widehat{x}}^{r-1} \widehat{u}+a(\eta, r) \partial_{\widehat{x}}^{r} \widehat{u}, \\
\partial_{\xi} \partial_{\eta}^{r-1} \widetilde{u}=\partial_{\xi}\left[\sum_{k=0}^{r-1}\left(\begin{array}{c}
r-1 \\
k
\end{array}\right) b(\xi, k)\left(\frac{1}{2}\right)^{r-k-1} \partial_{\widehat{x}}^{k} \partial_{\widehat{y}}^{r-k-1} \widehat{u}\right] \\
=\sum_{k=0}^{r-1}\left(\begin{array}{c}
r-1 \\
k
\end{array}\right)\left(\frac{1}{2}\right)^{r-k-1}\left[b(\xi, k+1) \partial_{\widehat{x}}^{k+1} \partial_{\widehat{y}}^{r-k-1} \widehat{u}\right. \\
\left.+\frac{b(\xi, k)}{2} \partial_{\widehat{x}}^{k} \partial_{\widehat{y}}^{r-k} \widehat{u}-\frac{k}{4} b(\xi, k-1) \partial_{\widehat{x}}^{k} \partial_{\widehat{y}}^{r-k-1} \widehat{u}\right] .
\end{gathered}
$$

Notice that for all $(\xi, \eta) \in \square$,

$$
0 \leq \frac{a(\eta, k)}{J_{0}(\xi, \eta)}=\left(\frac{1-\eta}{4}\right)^{k} \frac{8}{1-\eta}=2 a(\eta, k-1), \quad \forall k \geq 1
$$

so the following estimates hold

$$
\begin{aligned}
E_{r, \square}^{(1)}= & \left(\iint_{\square}\left(\partial_{\xi}^{r} \widetilde{u}\right)^{2}\left(1-\xi^{2}\right)^{r-1} d \xi d \eta\right)^{\frac{1}{2}}=\left\|2 a\left(\eta, \frac{2 r-1}{2}\right)\left(1-\xi^{2}\right)^{r-1} \partial_{\widehat{x}}^{r} \widehat{u}\right\|_{\triangle}, \\
E_{r, \square}^{(2)}= & \left(\iint_{\square}\left(\partial_{\eta}^{r} \widetilde{u}\right)^{2}\left(1-\eta^{2}\right)^{r-1} d \xi d \eta\right)^{\frac{1}{2}} \\
\leq & C \sum_{k=0}^{r}\left\|b(\xi, k)\left(1-\eta^{2}\right)^{\frac{r-2}{2}}(1+\eta)^{\frac{1}{2}} \partial_{\widehat{x}}^{k} \partial_{\widehat{y}}^{r-k} \widehat{u}\right\|_{\triangle} \\
E_{r, \square}^{(3)}= & \left(\iint_{\square}\left(1-\xi^{2}\right)^{r-2}\left(\partial_{\eta} \partial_{\xi}^{r-1} \widetilde{u}\right)^{2} d \xi d \eta\right)^{\frac{1}{2}} \\
\leq & C\left[\left\|\left(1-\xi^{2}\right)^{(r-2) / 2} a\left(\eta, \frac{2 r-5}{2}\right) \partial_{\widehat{x}}^{r-1} \widehat{u}\right\|_{\triangle}\right. \\
& \left.+\left\|\left(1-\xi^{2}\right)^{(r-2) / 2} a\left(\eta, \frac{2 r-1}{2}\right) \partial_{\widehat{x}}^{r} \widehat{u}\right\|_{\triangle}\right], \\
E_{r, \square}^{(4)}= & \left(\int_{\square}\left(1-\eta^{2}\right)^{r-2}\left(\partial_{\xi} \partial_{\eta}^{r-1} \widetilde{u}\right)^{2} d \xi d \eta\right)^{\frac{1}{2}} \\
\leq & C \sum_{k=0}^{r-1}\left[\left\|c(\eta, r) b(\xi, k+1) \partial_{\widehat{x}}^{k} \partial_{\widehat{y}}^{r-k-1} \widehat{u}\right\|_{\triangle}+\left\|c(\eta, r) b(\xi, k) \partial_{\widehat{x}}^{k} \partial_{\widehat{y}}^{r-k} \widehat{u}\right\|_{\triangle}\right. \\
& \left.+\left\|c(\eta, r) b(\xi, k-1) \partial_{\widehat{x}}^{k} \partial_{\widehat{y}}^{r-k-1} \widehat{u}\right\|_{\triangle}\right],
\end{aligned}
$$


where $c(\eta, r)=\left(1-\eta^{2}\right)^{(r-3) / 2}(1+\eta)^{1 / 2}$. For $r \geq 3$, using the boundedness of $a\left(\eta, \frac{2 r-5}{2}\right), c$ $(\eta, r), b(\xi, r-2)$, we obtain

$$
E_{r, \square}^{(1)}+E_{r, \square}^{(2)}+E_{r, \square}^{(3)}+E_{r, \square}^{(4)} \leq C\left(|\widehat{u}|_{r, \triangle}+|\widehat{u}|_{r-1, \triangle}\right) .
$$

A combination of (3.8a)-(3.9c) and (3.14b)-(3.14d) leads to the error estimate (3.7) for $\theta=0$.

Remark 3.1. For $0<\theta<1$, the integral

$$
\int_{\square} \frac{1}{J_{\theta}(\xi, \eta)} d \xi d \eta=16(1-\ln 2(1-\theta))-\frac{8}{1-\theta}[2 \theta(\ln 2 \theta-1)-2(\ln 2-1)]
$$

is finite. Thus, error estimate for $\left\|I_{p, \theta} \widehat{u}-\widehat{u}\right\|_{\mu, \triangle}, 0<\theta<1$ can be obtained using weighted norm $|\widehat{u}|_{2, \Delta}+\left\|\left(\partial_{\widehat{y}}-\partial_{\widehat{x}}\right)^{2} \widehat{u}\right\|_{J_{\theta}^{-1}, \Delta}+\|\widehat{\nabla} \widehat{u}\|_{J_{\theta}^{-1}, \Delta}$ for all $\widehat{u} \in H^{2}(\triangle)$ (ref. [23]). However, the error estimate for $\left\|\mathbb{I}_{p, 0} \widehat{u}-\widehat{u}\right\|_{\mu, \triangle}$ and $\left\|\mathbb{I}_{p, 1} \widehat{u}-\widehat{u}\right\|_{\mu, \Delta}$ requires $\widehat{u} \in H^{r}(\triangle), r \geq 3$.

Recall that $\mathscr{T}_{h}$ is a shape regular quasi-uniform mesh of the domain $\Omega$. There holds the following scaling results (cf. [7]).

Lemma 3.2. Let $K$ be an element in $\mathscr{T}_{h}$, and $F_{K}$ be the affine mapping from the reference triangle $\triangle$ to $K$. If $u \in H^{r}(\Omega), r \geq 0,\left.\widehat{u}\right|_{K}=u \circ F_{K}$, then

$$
|u|_{r, K} \leq C h^{1-r}|\widehat{u}|_{r, \Delta}, \quad|\widehat{u}|_{r, \Delta} \leq C h^{r-1}|u|_{r, K}
$$

The scaling argument leads to the following $h p$ error estimate.

Theorem 3.2. If $u \in H^{r}(\Omega), r \geq 3$, and $\mathscr{T}_{h}$ is a shape regular quasi-uniform mesh, then

$$
\left\|u-\mathscr{I}_{h, p}^{\theta} u\right\|_{\mu, \Omega} \leq C p^{\mu-r}\left(h^{r-\mu}|u|_{r, \Omega}+h^{r-\mu-1}|u|_{r-1, \Omega}\right), \quad \mu=0,1
$$

for $0 \leq \theta \leq 1$.

Proof. Recalling the definition (3.5) and then applying Lemma 3.2 and Theorem 3.1, we have

$$
\begin{gathered}
\quad\left\|u-\mathscr{I}_{h, p}^{\theta} u\right\|_{\mu, K}=\left\|\widehat{u} \circ F_{K}^{-1}-\left(\mathbb{I}_{p, \theta} \widehat{u}\right) \circ F_{K}^{-1}\right\|_{\mu, K} \leq C h^{1-\mu}\left\|\widehat{u}-\mathbb{I}_{p, \theta} \widehat{u}\right\|_{\mu, \Delta} \\
\leq C h^{1-\mu} p^{\mu-r}\left(|\widehat{u}|_{r, \Delta}+|\widehat{u}|_{r-1, \Delta}\right) \leq C p^{\mu-r}\left(h^{r-\mu}|u|_{r, K}+h^{r-\mu-1}|u|_{r-1, K}\right)
\end{gathered}
$$

for all $K \in \mathscr{T}_{h}$. Then the conclusion can be obtained by a direct summation. 


\section{Triangular spectral element methods in mixed formulation}

\subsection{The scheme}

Consider the elliptic boundary value problem

$$
-\nabla \cdot(\beta(\boldsymbol{x}) \nabla u)+\gamma(\boldsymbol{x}) u=f \quad \text { in } \Omega,\left.\quad u\right|_{\partial \Omega}=0,
$$

where $\Omega$ is an open, bounded and polygonal domain with Lipschitz boundary. We assume that $\beta(x, y) \gamma(x, y)$ are given positive functions in $\Omega$. By introducing an auxiliary variable $q$, the elliptic equation (4.1) is rewritten to the following first order system

$$
\begin{array}{ll}
\boldsymbol{q}-\beta \nabla u=0 & \text { in } \Omega, \\
-\nabla \cdot \boldsymbol{q}+\gamma u=f & \text { in } \Omega, \\
u=0 & \text { on } \partial \Omega .
\end{array}
$$

With the standard space $L^{2}(\Omega)$ and Sobolev spaces $H^{1}(\Omega), H_{0}^{1}(\Omega)$, the variational form of the problem (4.2) is of the form: find $(\boldsymbol{q}, u) \in\left(L^{2}(\Omega)\right)^{2} \times H_{0}^{1}(\Omega)$ such that

$$
\begin{aligned}
& (\boldsymbol{q}, \boldsymbol{v})-(\beta \nabla u, \boldsymbol{v})=0, \\
& (\boldsymbol{q}, \nabla w)+(\gamma u, w)=(f, w)
\end{aligned}
$$

for all $(\boldsymbol{v}, w) \in\left(L^{2}(\Omega)\right)^{2} \times H_{0}^{1}(\Omega)$.

Define

$$
V_{p, h}^{\theta}\left(\mathscr{T}_{h}\right)=\left\{\boldsymbol{v} \in\left(L^{2}(\Omega)\right)^{2}:\left.\boldsymbol{v}\right|_{K} \circ F_{K}^{-1} \in\left(Y_{p, \theta}(\triangle)\right)^{2}, \forall K \in \mathscr{T}_{h}\right\} .
$$

The spectral element formulation of (4.3) is to find $\left(\boldsymbol{Q}_{h, p}, U_{h, p}\right) \in V_{h, p}^{\theta}\left(\mathscr{T}_{h}\right) \times\left(S_{h, p}^{\theta}\left(\mathscr{T}_{h}\right) \cap\right.$ $H_{0}^{1}(\Omega)$ ), s.t.

$$
\begin{aligned}
& \left(\boldsymbol{Q}_{h, p}, \boldsymbol{v}_{h, p}\right)-\left(\beta \nabla U_{h, p}, \boldsymbol{v}_{h, p}\right)=0, \\
& \left(\boldsymbol{Q}_{h, p}, \nabla w_{h, p}\right)+\left(\gamma U_{h, p}, w_{h, p}\right)=\left(f, w_{h, p}\right)
\end{aligned}
$$

for all $\left(\boldsymbol{v}_{h, p}, w_{h, p}\right) \in V_{h, p}^{\theta}\left(\mathscr{T}_{h}\right) \times\left(S_{h, p}^{\theta}\left(\mathscr{T}_{h}\right) \cap H_{0}^{1}(\Omega)\right)$.

Theorem 4.1. Let $u$ and $U_{h, p}$ be the solutions of (4.3) and (4.5), respectively. If $u \in H_{0}^{1}(\Omega) \cap$ $H^{r}(\Omega)$ with $r>2$, then

$$
\left\|u-U_{h, p}\right\|_{\mu, \Omega} \leq C h^{r-\mu-1} p^{\mu-r}\|u\|_{r, \Omega}, \quad \mu=0,1,
$$

where $C$ is a positive constant independent of $h, p$ and $u$.

Proof. Set $\boldsymbol{v}=\nabla w$ in (4.3) and $\boldsymbol{v}_{h, p}=\nabla w_{h, p}$ in (4.5). The resulted equation implies

$$
\begin{array}{ll}
(\beta \nabla u, \nabla w)+(\gamma u, w)=(f, w), & \forall w \in H_{0}^{1}(\Omega), \\
\left(\beta \nabla U_{h, p}, \nabla w_{h, p}\right)+\left(\gamma U_{h, p}, w_{h, p}\right)=\left(f, w_{h, p}\right), & \forall w_{h, p} \in S_{h, p}^{\theta}\left(\mathscr{T}_{h}\right) \cap H_{0}^{1}(\Omega) .
\end{array}
$$


Taking $w=w_{h, p}$ in (4.7a) and then subtracting (4.7b) from the resulted equation, we have standard error equation

$$
\left.\left(\beta\left(\nabla u-\nabla U_{h, p}\right), \nabla w_{h, p}\right)+\left(\gamma\left(u-U_{h, p}\right), w_{h, p}\right)\right)=0, \quad \forall w_{h, p} \in S_{h, p}^{\theta}\left(\mathscr{T}_{h}\right) \cap H_{0}^{1}(\Omega) .
$$

The proof can be completed by using the standard error analysis technique with the interpolation estimate given by Theorem 3.2.

\subsection{Tensorial structure for efficient implementation}

Using the Lagrange nodal basis, we have

$$
\left.\boldsymbol{Q}_{h, p}\right|_{K_{j}}=\sum_{m=0}^{p} \sum_{n=0}^{p} \boldsymbol{Q}_{m n}^{(j)} \psi_{m n}\left(F_{K_{j}}^{-1}(x, y)\right),\left.\quad \boldsymbol{U}_{h, p}\right|_{K_{j}}=\sum_{m=0}^{p} \sum_{n=0}^{p} U_{m n}^{(j)} \psi_{m n}\left(F_{K_{j}}^{-1}(x, y)\right)
$$

for $\forall K_{j} \in \mathscr{T}_{h}$. Then the spectral element formulation (4.5) leads to the linear system

$$
\left[\begin{array}{lll}
\mathbb{M} & 0 & -\mathbb{C}_{x} \\
0 & \mathbb{M} & -\mathbb{C}_{y} \\
\mathbb{C}_{x}^{\mathrm{T}} & \mathbb{C}_{y}^{\mathrm{T}} & \mathbb{M}_{\gamma}
\end{array}\right]\left[\begin{array}{c}
\boldsymbol{Q}_{x} \\
\boldsymbol{Q}_{y} \\
\boldsymbol{U}
\end{array}\right]=\left[\begin{array}{l}
0 \\
0 \\
\boldsymbol{F}
\end{array}\right]
$$

where the global matrices $\mathbb{M}, \mathbb{C}_{x}, \mathbb{C}_{y}, \mathbb{M}_{\gamma}$ and righthand side $\boldsymbol{F}$ is obtained from the local contributions (or elements) $\mathbb{M}^{(j)}, \mathbb{C}_{x}^{(j)}, \mathbb{C}_{y}^{(j)}, \mathbb{M}_{\gamma}^{(j)}, \boldsymbol{F}^{(j)}$ by subassembly. The unknown vectors $\boldsymbol{Q}_{x}, \boldsymbol{Q}_{y}, \boldsymbol{U}$ consist of nodal values of $\boldsymbol{Q}_{h, p}$ and $U_{h, p}$. Since the basis functions are constructed by the tensor products of one-dimensional Lagrange basis functions $\left\{h_{m}(\xi), h_{n}(\eta)\right\}$, we expect to write the local matrices as the Kronecker products of one dimensional local matrices. Due to the usage of transform from rectangle to triangle, it is more complicate than that of the QSEM as presented below.

To fix the idea, we assume that $\beta(\boldsymbol{x})$ and $\gamma(\boldsymbol{x})$ are piecewise constants and $\beta(\boldsymbol{x})=$ $\beta_{j}, \gamma(\boldsymbol{x})=\gamma_{j}, \forall \boldsymbol{x} \in K_{j}$. Define matrices

$$
\widetilde{\mathbb{M}}=\left(\widetilde{M}_{i j}\right), \quad \widehat{\mathbb{M}}=\left(\widehat{M}_{i j}\right), \quad \widetilde{\mathbb{C}}=\left(\widetilde{C}_{i j}\right), \quad \widehat{\mathbb{C}}=\left(\widehat{C}_{i j}\right)
$$

with

$$
\begin{array}{ll}
\tilde{M}_{i j}=\int_{-1}^{1} h_{i}(\xi) h_{j}(\xi) d \xi, & \widehat{M}_{i j}=\int_{-1}^{1} h_{i}(\xi) h_{j}(\xi) \xi d \xi \\
\widetilde{C}_{i j}=\int_{-1}^{1} h_{i}(\xi) h_{j}^{\prime}(\xi) d \xi, & \widehat{C}_{i j}=\int_{-1}^{1} h_{i}(\xi) h_{j}^{\prime}(\xi) \xi d \xi
\end{array}
$$

and denote by

$$
\Psi_{m n}^{(j)}(x, y):=\psi_{m n}\left(F_{K_{j}}^{-1}(x, y)\right), \quad m, n=0,1, \cdots, p,
$$


i.e., the restrictions of typical basis functions to a given element $K_{j}$. Then

$$
\begin{aligned}
& \int_{K_{j}} \Psi_{m n}^{(j)}(x, y) \Psi_{m^{\prime} n^{\prime}}^{(j)}(x, y) d x d y \\
= & 2\left|K_{j}\right| \int_{\square} h_{m}(\xi) h_{n}(\eta) h_{m^{\prime}}(\xi) h_{n^{\prime}}(\eta) J_{\theta}(\xi, \eta) d \xi d \eta \\
= & \frac{\left|K_{j}\right|}{4}\left(\widetilde{M}_{m m^{\prime}} \tilde{M}_{n n^{\prime}}-\theta \widehat{M}_{m m^{\prime}} \tilde{M}_{n n^{\prime}}-(1-\theta) \widetilde{M}_{m m^{\prime}} \widehat{M}_{n n^{\prime}}\right) .
\end{aligned}
$$

where the expression (2.8) of $J_{\theta}$ is used. Similarly,

$$
\begin{aligned}
& \int_{K_{j}} \Psi_{m n}^{(j)}(x, y) \nabla \Psi_{m^{\prime} n^{\prime}}^{(j)}(x, y) d x d y \\
= & 2\left|K_{j}\right| \mathbb{J}_{F_{K_{j}}}^{-1} \int_{\square} h_{m}(\xi) h_{n}(\eta) J_{\theta} \mathbb{J}_{\theta}^{-1}\left[\begin{array}{c}
h_{m^{\prime}}^{\prime}(\xi) h_{n^{\prime}}(\eta) \\
h_{m^{\prime}}(\xi) h_{n^{\prime}}^{\prime}(\eta)
\end{array}\right] d \xi d \eta \\
= & \frac{\left|K_{j}\right|}{2} \mathbb{J}_{F_{K_{j}}}^{-1}\left[\begin{array}{c}
\left(-\theta \widehat{C}_{m m^{\prime}}+(2-\theta) \widetilde{C}_{m m^{\prime}}\right) \widetilde{M}_{n n^{\prime}}+\theta \widetilde{M}_{m m^{\prime}}\left(\widehat{C}_{n n^{\prime}}+\widetilde{C}_{n n^{\prime}}\right) \\
(1-\theta)\left(\widehat{C}_{m m^{\prime}}+\widetilde{C}_{m m^{\prime}}\right) \widetilde{M}_{n n^{\prime}}+\widetilde{M}_{m m^{\prime}}\left((\theta-1) \widehat{C}_{n n^{\prime}}+(1+\theta) \widetilde{C}_{n n^{\prime}}\right)
\end{array}\right],
\end{aligned}
$$

by the expression (2.8) and (2.10). With the formulations (4.13)-(4.14), the local contributions (or elements) $\mathbb{M}^{(j)}, \mathbb{M}_{\gamma}^{(j)}, \mathbb{C}_{x}^{(j)}, \mathbb{C}_{y}^{(j)}, \boldsymbol{F}^{(j)}$ can be written in Kronecker products as follows

$$
\begin{aligned}
& \mathbb{M}^{(j)}=\frac{\left|K_{j}\right|}{4}(\widetilde{\mathbb{M}} \otimes \widetilde{\mathbb{M}}-\theta(\widehat{\mathbb{M}} \otimes \widetilde{\mathbb{M}}-\widetilde{\mathbb{M}} \otimes \widehat{\mathbb{M}})-\widetilde{\mathbb{M}} \otimes \widehat{\mathbb{M}}), \\
& \mathbb{C}_{x}^{(j)}=\frac{\left|K_{j}\right|}{2}\left[\left(\tau_{11}^{(j)} \widehat{\mathbb{C}}+\tau_{12}^{(j)} \widetilde{\mathbb{C}}\right) \otimes \widetilde{\mathbb{M}}+\widetilde{\mathbb{M}} \otimes\left(\tau_{13}^{(j)} \widehat{\mathbb{C}}+\tau_{14}^{(j)} \widetilde{\mathbb{C}}\right)\right], \\
& \mathbb{C}_{y}^{(j)}=\frac{\left|K_{j}\right|}{2}\left[\left(\tau_{21}^{(j)} \widehat{\mathbb{C}}+\tau_{22}^{(j)} \widetilde{\mathbb{C}}\right) \otimes \widetilde{\mathbb{M}}+\widetilde{\mathbb{M}} \otimes\left(\tau_{23}^{(j)} \widehat{\mathbb{C}}+\tau_{24}^{(j)} \widetilde{\mathbb{C}}\right)\right], \\
& \mathbb{M}_{\gamma}^{(j)}=\gamma_{j} \mathbb{M}^{(j)}, \quad F^{(j)}=\mathbb{M}^{(j)} \boldsymbol{f},
\end{aligned}
$$

where $\boldsymbol{f}$ is the vector of interpolant nodal values of $f(\boldsymbol{x})$ and

$$
\left(\tau_{i j}^{(j)}\right)_{2 \times 4}=\mathbb{J}_{F_{K_{j}}}^{-1}\left[\begin{array}{cccc}
-\theta & 2-\theta & \theta & \theta \\
1-\theta & 1-\theta & \theta-1 & 1+\theta
\end{array}\right] .
$$

The formulation for $\boldsymbol{F}^{(j)}$ results from the insertion of the interpolant of $f(\boldsymbol{x})$ into the right handside integration.

Remark 4.1. For general variable coefficients $\beta(\boldsymbol{x}), \gamma(\boldsymbol{x})$, the coefficients are firstly approximated by LGL interpolation, see more details in [8]. 
The main advantage of the proposed triangular spectral element mixed formulation is that it does not involve the stiffness matrix which consists of integral

$$
\begin{aligned}
& \iint_{K_{j}} \nabla \Psi_{m n}^{(j)} \cdot \nabla \Psi_{m^{\prime} n^{\prime}}^{(j)} \mathrm{d} \widehat{x} \mathrm{~d} \widehat{y} \\
= & 2\left|K_{j}\right| \iint_{\square}\left(\mathbb{J}_{F_{K_{j}}}^{-1} \mathbb{J}_{\theta}^{-1} \widetilde{\nabla} \psi_{m n}\right) \cdot\left(J_{\theta} \mathbb{J}_{F_{K_{j}}}^{-1} \mathbb{J}_{\theta}^{-1} \widetilde{\nabla} \psi_{m^{\prime} n^{\prime}}\right) \mathrm{d} \xi \mathrm{d} \eta,
\end{aligned}
$$

where $\tilde{\nabla}=\left(\partial_{\xi}, \partial_{\eta}\right)^{\mathrm{T}}$. Recalling (2.10), $\mathbb{J}_{\theta}^{-1}$ has singularities in $\square$ and the singularity can be removed by multiplying $J_{\theta}$. Therefore, the LGL quadrature can be used to calculate integrals (4.13) and (4.14) but can not be used to calculate integral (4.17). Some analytic means for the computation of (4.17) have been intensively studied by using mode basis (cf. [23]). However, it seems to be not applicable to the case with general variable coefficients.

Here, LGL quadrature is used to calculate the entries $\widetilde{M}_{i j}, \widehat{M}_{i j}, \widetilde{C}_{i j}, \widehat{C}_{i j}$. For example, we actually set

$$
\widetilde{M}_{i j}=\sum_{k=0}^{N} h_{i}\left(\xi_{k}\right) h_{j}\left(\xi_{k}\right) \omega_{k}, \quad \widehat{M}_{i j}=\sum_{k=0}^{N} h_{i}\left(\xi_{k}\right) h_{j}\left(\xi_{k}\right) \xi_{k} \omega_{k}
$$

in the numerical calculation. Since the nodal basis with respect to LGL points is adopted, $\widetilde{\mathbb{M}}$ and $\widehat{\mathbb{M}}$ become diagonal, i.e., $\widetilde{\mathbb{M}}=\operatorname{diag}\left(\omega_{k}\right)$ and $\widehat{\mathbb{M}}=\operatorname{diag}\left(\xi_{k} \omega_{k}\right)$. Together with the Kronecker product formulation, we conclude that $\mathbb{M}$ and $\mathbb{M}_{\gamma}$ are diagonal too. Therefore $\boldsymbol{Q}_{x}, \boldsymbol{Q}_{y}$ can be easily solved from $\boldsymbol{U}$ and the linear system (4.10) reduces to

$$
\left(\mathbb{C}_{x}^{\mathrm{T}} \mathbb{M}^{-1} \mathbb{C}_{x}+\mathbb{C}_{y}^{\mathrm{T}} \mathbb{M}^{-1} \mathbb{C}_{y}+\mathbb{M}_{\gamma}\right) \boldsymbol{U}=\boldsymbol{F} .
$$

Noting that $\widetilde{M}_{p p}=\widehat{M}_{p p}$ for $\xi_{p}=1$, there are zero entries on the diagonal of $\mathbb{M}^{(j)}$ due to the usage of LGL quadrature. In particular for the two typical cases, we have

$$
\mathbb{M}^{(j)}= \begin{cases}\frac{\left|K_{j}\right|}{4} \widetilde{\mathbb{M}} \otimes(\widetilde{\mathbb{M}}-\widehat{\mathbb{M}}), & \theta=0, \\ \frac{\left|K_{j}\right|}{4}\left(\widetilde{\mathbb{M}} \otimes \widetilde{\mathbb{M}}-\frac{1}{2} \widehat{\mathbb{M}} \otimes \widetilde{\mathbb{M}}-\frac{1}{2} \widetilde{\mathbb{M}} \otimes \widehat{\mathbb{M}}\right), & \theta=\frac{1}{2} .\end{cases}
$$

It is not difficult to find out that $\mathbb{M}^{(j)}$ has $p+1$ zero entries on the diagonal when $\theta=0$ and has only one zero entry on the diagonal in the case $\theta=\frac{1}{2}$. The existance of zero entries on the diagonal makes mass matrix $\mathbb{M}$ singular. Therefore the linear system (4.19) is not available if LGL quadrature with $p+1$ points is used for the calculation of all entries of $\widehat{\mathbb{M}}$. It is evidence that

$$
\int_{-1}^{1} h_{p}^{2}(\xi) d \xi \neq \int_{-1}^{1} h_{p}^{2}(\xi) \xi d \xi
$$

Therefore, $\widetilde{M}_{p p}=\widehat{M}_{p p}$ is due to the fact that resolution of $p+1$ points LGL quadrature is not enough to distinguish these two integrals. In order to overcome this difficulty, we use Legendre-Gauss quadrature with $p+1$ quadrature points for the special entry $\widehat{M}_{p p}$. By improving the accuracy of $\widehat{M}_{p p}$, the revised approximated mass matrix $\mathbb{M}^{(j)}$ is not singular and the linear system (4.19) is available. 


\subsection{Numerical results}

Now, we present some numerical examples to verify the accuracy of the proposed TSEMs using two typical $\triangle \mapsto \square$ transforms (2.11) and (2.12). Both convergence rates against polynomial degree $p$ and mesh size $h$ are presented. Complex domains with unstructured meshes and non-smooth solutions are considered.

In all numerical experiments, we test the elliptic problem (4.1) on various domains with $\beta=e^{x+y}, \gamma=1$. Smooth exact solution:

$$
u(x, y)=\cos \left(\pi\left(x^{2}+y^{2}\right)\right)
$$

and non-smooth exact solutions:

$$
\begin{aligned}
& u(x, y)=(x+y)^{\frac{5}{2}} \\
& u(x, y)=(x-y)^{\frac{8}{3}}\left(e^{x y}+1\right)
\end{aligned}
$$

are tested.

Hereafter, we denote by $E_{p}$ the $L^{2}$-error of the numerical solution for fixed mesh and polynomial degree $p, E_{h}$ the $L^{2}$-error for fixed polynomial degree and mesh size $h$. All $L^{2}$ errors are calculated by using much higher order Gauss quadrature element-by-element. The convergence rates against $p$ are defined as

$$
-\frac{\ln E_{p_{k}}-\ln E_{p_{k+1}}}{p_{k}-p_{k+1}}, \quad-\frac{\ln E_{p_{k}}-\ln E_{p_{k+1}}}{\ln p_{k}-\ln p_{k+1}}
$$

which are the constants $c$ and $r$ in the expected convergence rates $\mathscr{O}\left(e^{-c p}\right)$ and $\mathscr{O}\left(p^{-r}\right)$ for smooth and non-smooth solutions, respectively. The convergence rate against $h$ is defined as

$$
\frac{\ln E_{h_{k}}-\ln E_{h_{k+1}}}{\ln h_{k}-\ln h_{k+1}} .
$$

Example 4.1. We first test the accuracy of the proposed TSEMs by compared with QSEM on Cartesian mesh. For this purpose, we set $\Omega=[0,1]^{2}$ and the triangular meshes are generated by subdividing each element in the corresponding Cartesian meshes into two tringles, see Fig. 5 for the initial meshes and corresponding LGL-sepctral element nodes distributions.

In this case, solution (4.22a) and (4.22b) have point and line singularity in the computational domain $\Omega$ and belong to $H^{4-\alpha}$ and $H^{3-\beta}$, respectively. $L^{2}$-errors and corresponding convergence rates presented in Tables 4.1 and 4.1, and Fig. 6 show that QSEM and the proposed TSEMs share very similar convergence behavior. For smooth solution (4.21) all their errors decay like $\mathscr{O}\left(e^{-c p}\right)$ for fixed mesh. Spectral accuracy is obtained for smooth solution and optimal convergence rate is observed for non-smooth solutions. It is worthy to point out that the obtained results also show that the TSEM using $T_{1 / 2}$ is more stable than that using $T_{0}$. 


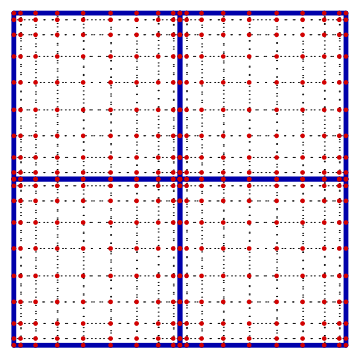

(a) affine mapping

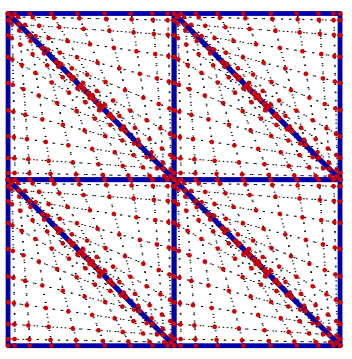

(b) mapping $T_{1 / 2}$

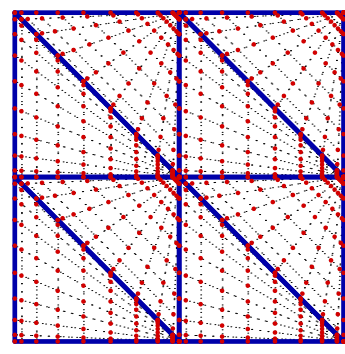

(c) mapping $T_{0}$

Figure 5: Initial Cartesian and triangular meshes and LGL-spectral element nodes distribution.

Table 1: $L^{2}$-errors and convergence rates against $p$ for fixed mesh.

\begin{tabular}{|c|c|c|c|c|c|c|c|}
\hline & \multirow{2}{*}{$p$} & \multicolumn{2}{|c|}{ SEM } & \multicolumn{2}{|c|}{ TSEM using $T_{0}$} & \multicolumn{2}{|c|}{ TSEM using $T_{1 / 2}$} \\
\hline & & error & rate & error & rate & error & rate \\
\hline \multirow{4}{*}{ smooth solution } & 4 & $2.218 \mathrm{E}-03$ & & $1.482 \mathrm{E}-03$ & & $9.440 \mathrm{E}-04$ & \\
\hline & 8 & $1.010 \mathrm{E}-06$ & 1.924 & $7.168 \mathrm{E}-07$ & 1.908 & $4.306 \mathrm{E}-07$ & 1.923 \\
\hline & 12 & $1.340 \mathrm{E}-10$ & 2.232 & $1.796 \mathrm{E}-10$ & 2.073 & $4.686 \mathrm{E}-11$ & 2.281 \\
\hline & 16 & $8.716 \mathrm{E}-15$ & 2.410 & $4.430 \mathrm{E}-14$ & 2.077 & 4.049E-14 & 1.763 \\
\hline \multirow{5}{*}{$\begin{array}{l}\text { non-smooth solution } \\
\text { (4.22a) }\end{array}$} & 4 & $9.142 \mathrm{E}-06$ & & $1.130 \mathrm{E}-05$ & & $7.274 \mathrm{E}-06$ & \\
\hline & 8 & $9.867 \mathrm{E}-08$ & 6.534 & $8.855 \mathrm{E}-08$ & 6.996 & $8.888 \mathrm{E}-08$ & 6.355 \\
\hline & 12 & $6.961 \mathrm{E}-09$ & 6.539 & $6.485 \mathrm{E}-09$ & 6.447 & $6.499 \mathrm{E}-09$ & 6.451 \\
\hline & 16 & 1.037E-09 & 6.618 & $9.833 \mathrm{E}-10$ & 6.557 & $9.846 \mathrm{E}-10$ & 6.560 \\
\hline & 20 & $2.337 \mathrm{E}-10$ & 6.678 & $2.238 \mathrm{E}-10$ & 6.632 & $2.241 \mathrm{E}-10$ & 6.634 \\
\hline \multirow{5}{*}{$\begin{array}{c}\text { non-smooth solution } \\
(4.22 \mathrm{~b})\end{array}$} & 4 & $3.215 \mathrm{E}-04$ & & $1.722 \mathrm{E}-03$ & & $4.244 \mathrm{E}-04$ & \\
\hline & 8 & $2.803 \mathrm{E}-05$ & 3.520 & $1.361 \mathrm{E}-04$ & 3.661 & $3.203 \mathrm{E}-05$ & 3.728 \\
\hline & 12 & $7.411 \mathrm{E}-06$ & 3.281 & $3.620 \mathrm{E}-05$ & 3.267 & $8.089 \mathrm{E}-06$ & 3.394 \\
\hline & 16 & $2.926 \mathrm{E}-06$ & 3.230 & $1.436 \mathrm{E}-05$ & 3.213 & $3.124 \mathrm{E}-06$ & 3.307 \\
\hline & 20 & $1.430 \mathrm{E}-06$ & 3.209 & 7.045E-06 & 3.193 & 1.507E-06 & 3.267 \\
\hline
\end{tabular}

Example 4.2. This example is to show the feasibility and accuracy of the proposed TSEMs on complex domains with mixed and unstructured meshes. We consider two polygonal domains: polygon A and polygon B with vertices given by:

$$
\begin{aligned}
\text { polygon A: } & (0,0),(1,0),\left(2, \frac{1}{2}\right),(1,1),\left(1, \frac{1}{2}\right),(0,1) \\
\text { polygon B : } & R\left(\cos \theta_{k}+\cos \frac{\pi}{8}, \sin \theta_{k}+\sin \frac{\pi}{8}\right), \\
& R\left(\cos \theta_{k}+3 \cos \frac{\pi}{8}+3 \sin \frac{\pi}{8}, \sin \theta_{k}+\sin \frac{\pi}{8}\right), \quad k=0,1, \cdots, 7,
\end{aligned}
$$

where $\theta_{k}=\frac{2 k+1}{8} \pi, R=\frac{1}{2} \cos \frac{\pi}{8}$. Polygon A is triangulated by hybrid meshes (with both rectangle and triangle elements) and polygon $\mathrm{B}$ is triangulated by unstructured triangular meshes, see Fig. 7 for initial meshes and spectral element nodes distributions. Solution (4.22a) and (4.22b) also have point and line singularity in the computational domain $\Omega$ and belong to $H^{4-\alpha}$ and $H^{3-\beta}$, respectively. The hybrid meshes used here are essentially 


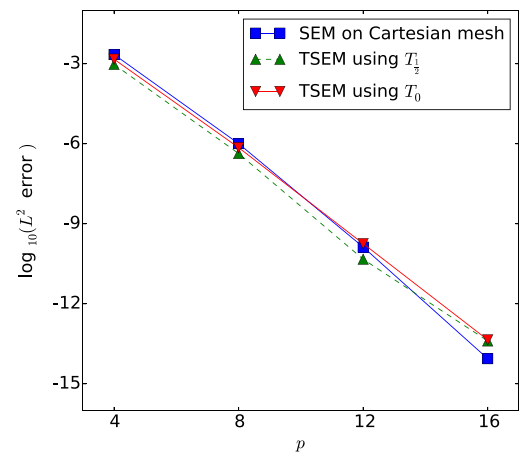

(a) smooth solution

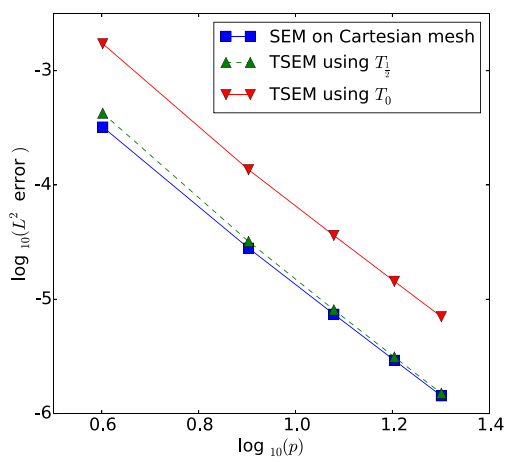

(c) non-smooth solution (4.22b)

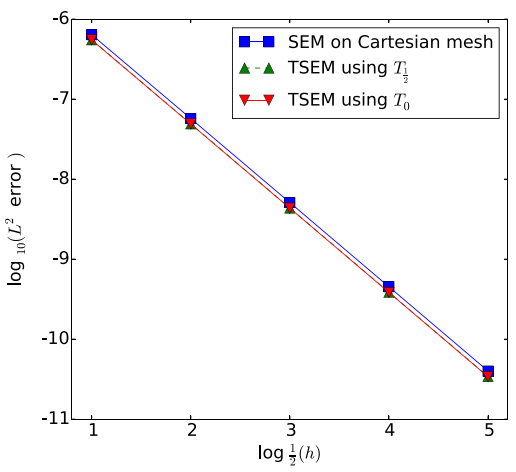

(e) non-smooth solution $(4.22 \mathrm{a})$

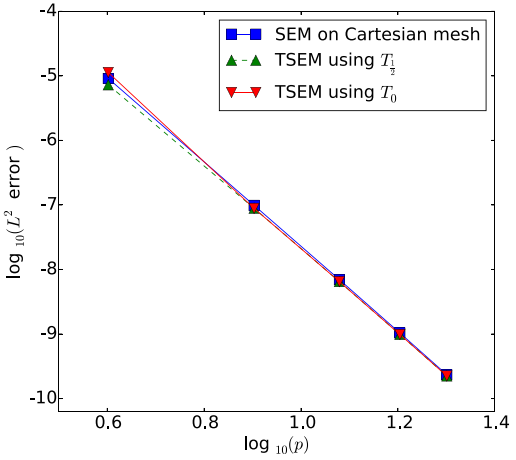

(b) non-smooth solution (4.22a)

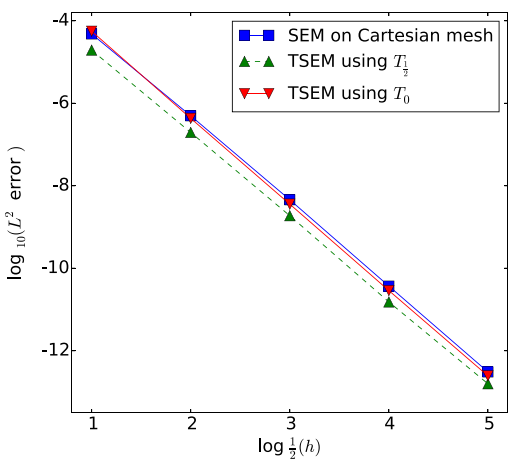

(d) smooth solution

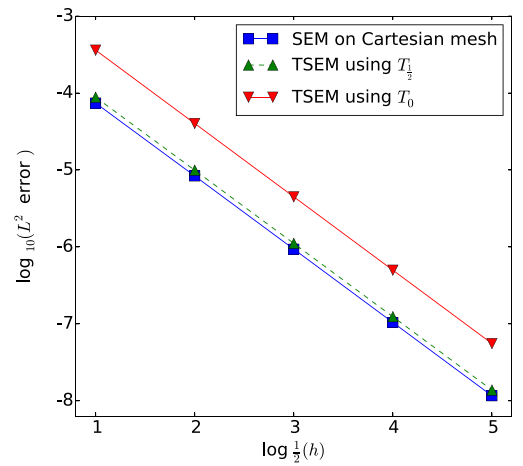

(f) non-smooth solution (4.22b)

Figure 6: Comparison of numerical errors between QSEM and TSEMs ((a), (b), (c): errors against $p$; (d), (e), (f): errors against $h$ ).

different with that investigated in [17]. Their hybrid mesh actually has a hanging vertex. The intersection of the triangular element with the neighboring rectangular element is not an entire edge of the mesh. All hybrid meshes used in this paper are conformal without 


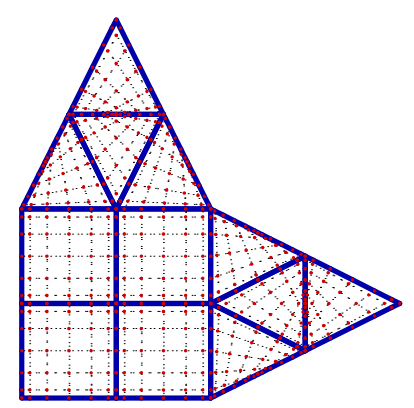

(a) Polygonal A

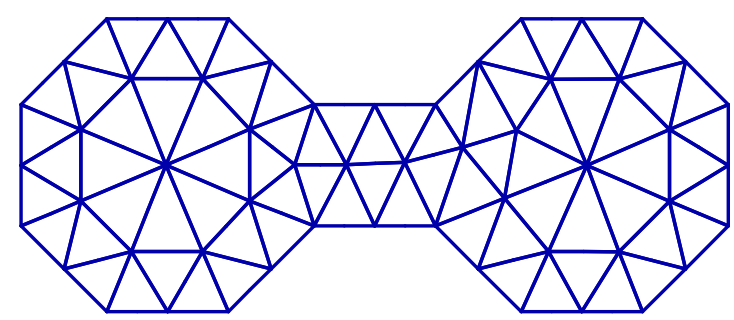

(b) Polygonal B

Figure 7: Polygonal domains with mixed and unstructured meshes.

Table 2: $L^{2}$-errors and convergence rates against $h$ for fixed $p=6$.

\begin{tabular}{|c|c|c|c|c|c|c|c|}
\hline & \multirow{2}{*}{$h$} & \multicolumn{2}{|c|}{ SEM } & \multicolumn{2}{|c|}{ TSEM using $T_{0}$} & \multicolumn{2}{|c|}{ TSEM using $T_{1 / 2}$} \\
\hline & & error & rate & error & rate & error & rate \\
\hline \multirow{5}{*}{ smooth solution } & & 4.795E-05 & & $5.587 \mathrm{E}-05$ & & $1.945 \mathrm{E}-05$ & \\
\hline & & $4.967 \mathrm{E}-07$ & 6.593 & $4.315 \mathrm{E}-07$ & 7.016 & $1.962 \mathrm{E}-07$ & 6.631 \\
\hline & & $4.571 \mathrm{E}-09$ & 6.764 & $3.582 \mathrm{E}-09$ & 6.912 & $1.877 \mathrm{E}-09$ & 6.708 \\
\hline & 16 & $3.691 \mathrm{E}-11$ & 6.952 & $2.878 \mathrm{E}-11$ & 6.960 & $1.523 \mathrm{E}-11$ & 6.946 \\
\hline & $\frac{1}{32}$ & $3.061 \mathrm{E}-13$ & 6.914 & $2.459 \mathrm{E}-13$ & 6.870 & $1.580 \mathrm{E}-13$ & 6.591 \\
\hline \multirow{5}{*}{$\begin{array}{l}\text { non-smooth solution } \\
(4.22 \mathrm{a})\end{array}$} & & $6.378 \mathrm{E}-07$ & & 5.491E-07 & & $5.518 \mathrm{E}-07$ & \\
\hline & $\frac{1}{4}$ & $5.716 \mathrm{E}-08$ & 3.480 & $4.885 \mathrm{E}-08$ & 3.491 & 4.914E-08 & 3.489 \\
\hline & $\frac{1}{8}$ & 5.089E-09 & 3.490 & 4.332E-09 & 3.495 & 4.359E-09 & 3.495 \\
\hline & $\frac{1}{16}$ & $4.514 \mathrm{E}-10$ & 3.495 & $3.835 \mathrm{E}-10$ & 3.498 & $3.860 \mathrm{E}-10$ & 3.497 \\
\hline & $\frac{1}{32}$ & $3.998 \mathrm{E}-11$ & 3.497 & $3.393 \mathrm{E}-11$ & 3.499 & $3.415 \mathrm{E}-11$ & 3.499 \\
\hline \multirow{5}{*}{$\begin{array}{l}\text { non-smooth solution } \\
(4.22 \mathrm{~b})\end{array}$} & & 7.407E-05 & & $3.611 \mathrm{E}-04$ & & $8.822 \mathrm{E}-05$ & \\
\hline & 4 & $8.366 \mathrm{E}-06$ & 3.146 & $4.023 \mathrm{E}-05$ & 3.166 & 9.939E-06 & 3.150 \\
\hline & & $9.352 \mathrm{E}-07$ & 3.161 & $4.480 \mathrm{E}-06$ & 3.167 & $1.110 \mathrm{E}-06$ & 3.162 \\
\hline & $\frac{1}{16}$ & $1.042 \mathrm{E}-07$ & 3.165 & $4.986 \mathrm{E}-07$ & 3.168 & $1.237 \mathrm{E}-07$ & 3.166 \\
\hline & $\frac{1}{32}$ & $1.161 \mathrm{E}-08$ & 3.166 & $5.551 \mathrm{E}-08$ & 3.167 & $1.378 \mathrm{E}-08$ & 3.166 \\
\hline
\end{tabular}

any hanging points. That is the reason why we can have optimal convergence rates here. $L^{2}$-errors and corresponding convergence rates against polynomial degree $p$ and mesh size $h$ are presented in Tables 4.1-4.3 and Figs. 8-9. For smooth solution (4.21), errors using different TSEMs decay like $\mathscr{O}\left(e^{-c p}\right)$ for fixed mesh, i.e., spectral accuracy is obtained. On the other hand, optimal convergence rates are observed for non-smooth solutions. The TSEM using $T_{1 / 2}$ shows some advantages over that using $T_{0}$ in stability (see. Fig. 9: (c), (f)). We make a comparison between the proposed TSEM and the TSEM presented in [23]. $L^{2}$-errors and convergence rates presented in Table 4.3 and Table 4.3 show that they share similar accuracy performance. Table 4.3 compares the condition numbers of corresponding resulted linear systems. Although TSEM using $T_{1 / 2}$ has larger conditon number they are in the same order with respect to $p$. 
Table 3: $L^{2}$-errors and convergence rates against $p$ for fixed mesh (mixed mesh).

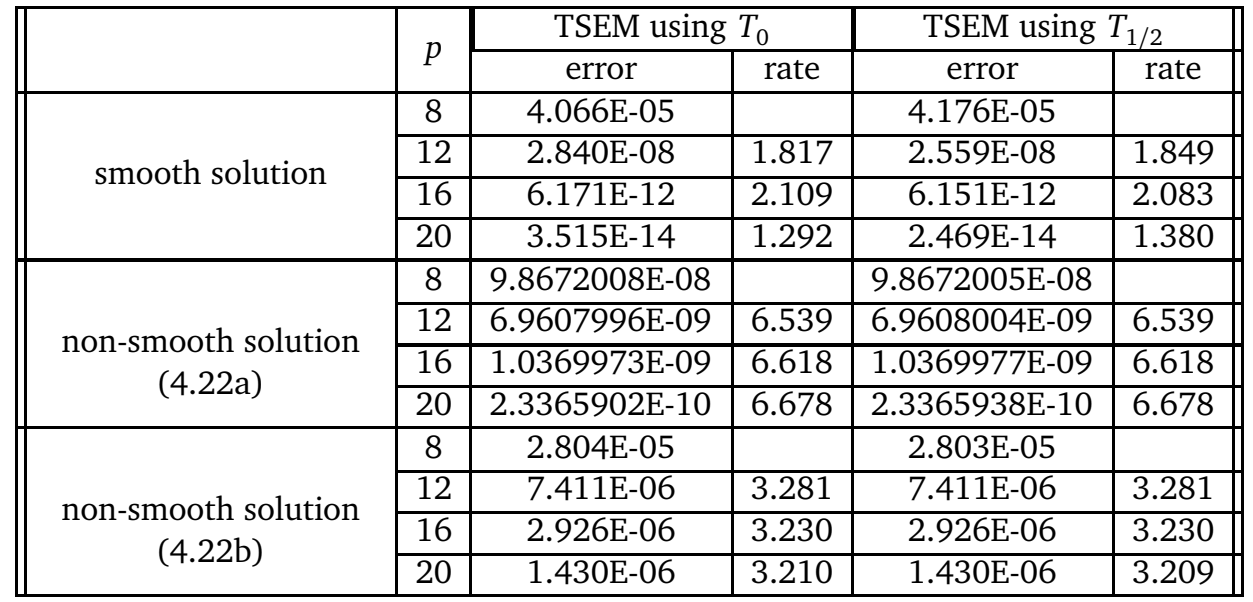

Table 4: $L^{2}$-errors and convergence rates against $h$ for fixed $p=6$ (mixed mesh).

\begin{tabular}{|c|c|c|c|c|c|}
\hline & \multirow{2}{*}{$h$} & \multicolumn{2}{|c|}{ TSEM using $T_{0}$} & \multicolumn{2}{|c|}{ TSEM using $T_{1 / 2}$} \\
\hline & & error & rate & error & rate \\
\hline \multirow{4}{*}{ smooth solution } & $\frac{1}{4}$ & $1.370 \mathrm{E}-05$ & & $1.324 \mathrm{E}-05$ & \\
\hline & $\frac{1}{8}$ & 9.063E-08 & 7.240 & 8.685E-08 & 7.252 \\
\hline & $\frac{1}{16}$ & $7.253 \mathrm{E}-10$ & 6.965 & $6.841 \mathrm{E}-10$ & 6.988 \\
\hline & $\frac{1}{32}$ & $5.681 \mathrm{E}-12$ & 6.996 & $5.334 \mathrm{E}-12$ & 7.003 \\
\hline \multirow{4}{*}{$\begin{array}{l}\text { non-smooth solution } \\
\qquad(4.22 \mathrm{a})\end{array}$} & $\frac{1}{4}$ & 5.71630E-08 & & 5.71618E-08 & \\
\hline & $\frac{1}{8}$ & 5.08885E-09 & 3.490 & 5.08885E-09 & 3.490 \\
\hline & $\frac{1}{16}$ & $4.51442 \mathrm{E}-10$ & 3.495 & $4.51442 \mathrm{E}-10$ & 3.495 \\
\hline & $\frac{1}{32}$ & $4.01016 \mathrm{E}-11$ & 3.493 & 3.99959E-11 & 3.497 \\
\hline \multirow{4}{*}{$\begin{array}{l}\text { non-smooth solution } \\
\qquad(4.22 \mathrm{~b})\end{array}$} & $\frac{1}{4}$ & $8.37314 \mathrm{E}-06$ & & $8.36542 \mathrm{E}-06$ & \\
\hline & $\frac{1}{8}$ & 9.35612E-07 & 3.162 & $9.35175 \mathrm{E}-07$ & 3.161 \\
\hline & $\frac{1}{16}$ & 1.04269E-07 & 3.166 & $1.04245 \mathrm{E}-07$ & 3.165 \\
\hline & $\frac{1}{32}$ & $1.16131 \mathrm{E}-08$ & 3.166 & $1.16117 \mathrm{E}-08$ & 3.166 \\
\hline
\end{tabular}

\subsection{Concluding remarks and future work}

In this paper, we have introduced a new triangular spectral element method for unstructured mesh by using a nodal basis and rectangle-triangle transforms. It is a mixed formulation and fully enjoys the tensorial product property and flexibility in handling complex domains. Due to the exemption of the calculation of stiffness matrix, no singularity appears in the generation of the discrete system. An $h p$ priori error estimate was presented for the proposed method. The results obtained by this mixed formulation is a good evidence that discontinuous Galerkin methods based on mixed formulation (e.g., LDG, HDG) can be used to handle the singularity and node inconsistency simultaneously. The development of discontinuous triangular spectral element method based on mixed formulation will be our future work. 
Table 5: $L^{2}$-errors and convergence rates against $p$ for fixed mesh (unstructured triangular mesh).

\begin{tabular}{||c|c|c|c|c|c|c|c||}
\hline \multirow{4}{*}{ smooth solution } & \multirow{2}{*}{$p$} & \multicolumn{2}{|c|}{ TSEM using $T_{0}$} & \multicolumn{2}{c|}{ TSEM using $T_{1 / 2}$} & \multicolumn{2}{c||}{ TSEM in [23] } \\
\cline { 2 - 8 } & 8 & $5.225 \mathrm{E}-05$ & 1.569 & $1.115 \mathrm{E}-05$ & 1.794 & $9.264 \mathrm{E}-06$ & 1.784 \\
\cline { 2 - 8 } & 12 & $2.735 \mathrm{E}-08$ & 1.889 & $1.391 \mathrm{E}-08$ & 1.672 & $1.319 \mathrm{E}-08$ & 1.639 \\
\cline { 2 - 8 } & 16 & $3.076 \mathrm{E}-12$ & 2.273 & $2.097 \mathrm{E}-12$ & 2.200 & $2.095 \mathrm{E}-12$ & 2.187 \\
\cline { 2 - 8 } & 20 & $6.809 \mathrm{E}-13$ & 0.377 & $2.620 \mathrm{E}-13$ & 0.520 & $1.686 \mathrm{E}-13$ & 0.630 \\
\hline \multirow{4}{*}{$\begin{array}{c}\text { non-smooth solution } \\
\text { (4.22a) }\end{array}$} & 8 & $8.746 \mathrm{E}-08$ & 7.058 & $9.294 \mathrm{E}-08$ & 5.700 & $9.248 \mathrm{E}-08$ & 5.484 \\
\cline { 2 - 8 } & 12 & $8.938 \mathrm{E}-09$ & 5.625 & $1.051 \mathrm{E}-08$ & 5.376 & $1.052 \mathrm{E}-08$ & 5.360 \\
\cline { 2 - 8 } & 16 & $1.844 \mathrm{E}-09$ & 5.563 & $2.242 \mathrm{E}-09$ & 5.371 & $2.253 \mathrm{E}-09$ & 5.358 \\
\cline { 2 - 8 } non-smooth solution & 20 & $5.254 \mathrm{E}-10$ & 5.527 & $6.748 \mathrm{E}-10$ & 5.380 & $6.800 \mathrm{E}-10$ & 5.368 \\
\hline (4.22b) & 8 & $1.472 \mathrm{E}-05$ & 4.450 & $1.111 \mathrm{E}-05$ & 3.587 & $1.118 \mathrm{E}-05$ & 3.301 \\
\cline { 2 - 8 } & 12 & $3.412 \mathrm{E}-06$ & 3.606 & $2.816 \mathrm{E}-06$ & 3.385 & $2.919 \mathrm{E}-06$ & 3.312 \\
\cline { 2 - 8 } & 16 & $1.619 \mathrm{E}-06$ & 2.591 & $1.170 \mathrm{E}-06$ & 3.054 & $1.156 \mathrm{E}-06$ & 3.219 \\
\cline { 2 - 7 } & 20 & $7.574 \mathrm{E}-07$ & 3.405 & $5.781 \mathrm{E}-07$ & 3.159 & $6.042 \mathrm{E}-07$ & 2.908 \\
\hline
\end{tabular}

Table 6: $L^{2}$-errors and convergence rates against $h$ for fixed $p=6$ (unstructured triangular mesh).

\begin{tabular}{|c|c|c|c|c|c|c|c|}
\hline & \multirow{2}{*}{$h$} & \multicolumn{2}{|c|}{ TSEM using $T_{0}$} & \multicolumn{2}{|c|}{ TSEM using $T_{1 / 2}$} & \multicolumn{2}{|c|}{ TSEM in [23] } \\
\hline & & error & rate & error & rate & error & rate \\
\hline \multirow{4}{*}{ smooth solution } & $\frac{1}{4}$ & 1.409E-05 & 6.292 & $9.948 \mathrm{E}-06$ & 5.306 & 9.505E-06 & 5.297 \\
\hline & $\frac{1}{8}$ & $1.082 \mathrm{E}-07$ & 7.024 & 7.039E-08 & 7.143 & $6.746 \mathrm{E}-08$ & 7.138 \\
\hline & $\frac{1}{16}$ & $8.179 \mathrm{E}-10$ & 7.048 & $5.526 \mathrm{E}-10$ & 6.993 & $5.322 \mathrm{E}-10$ & 6.986 \\
\hline & $\frac{10}{\frac{1}{32}}$ & $6.367 \mathrm{E}-12$ & 7.005 & $4.350 \mathrm{E}-12$ & 6.989 & $4.205 \mathrm{E}-12$ & 6.984 \\
\hline \multirow{4}{*}{$\begin{array}{l}\text { non-smooth solution } \\
\qquad(4.22 \mathrm{a})\end{array}$} & $\frac{1}{4}$ & 7.120E-08 & 2.665 & 5.524E-08 & 2.999 & 5.472E-08 & 2.998 \\
\hline & $\frac{1}{8}$ & 7.933E-09 & 3.166 & $6.911 \mathrm{E}-09$ & 2.999 & $6.848 \mathrm{E}-09$ & 2.998 \\
\hline & $\frac{8}{16}$ & $1.046 \mathrm{E}-09$ & 2.922 & $8.643 \mathrm{E}-10$ & 2.999 & $8.567 \mathrm{E}-10$ & 2.999 \\
\hline & $\frac{10}{32}$ & $1.273 \mathrm{E}-10$ & 3.039 & $1.089 \mathrm{E}-10$ & 2.988 & $1.073 \mathrm{E}-10$ & 2.997 \\
\hline \multirow{4}{*}{$\begin{array}{l}\text { non-smooth solution } \\
\qquad(4.22 \mathrm{~b})\end{array}$} & $\frac{1}{4}$ & $3.136 \mathrm{E}-06$ & 3.225 & $2.689 \mathrm{E}-06$ & 3.204 & $2.643 \mathrm{E}-06$ & 3.199 \\
\hline & $\frac{1}{8}$ & $3.286 \mathrm{E}-07$ & 3.255 & $2.846 \mathrm{E}-07$ & 3.240 & $2.793 \mathrm{E}-07$ & 3.242 \\
\hline & $\frac{1}{16}$ & 4.782E-08 & 2.781 & $3.986 \mathrm{E}-08$ & 2.836 & $3.958 \mathrm{E}-08$ & 2.819 \\
\hline & $\frac{10}{\frac{1}{32}}$ & $3.601 \mathrm{E}-09$ & 3.731 & 2.813E-09 & 3.825 & $3.008 \mathrm{E}-09$ & 3.718 \\
\hline
\end{tabular}

Table 7: A Comparison of condition numbers of TSEM using $T_{1 / 2}$ and TSEM presented in [23] (unstructured triangular mesh).

\begin{tabular}{||c|c|c|l||}
\hline$p$ & TSEM in $[23]$ & TSEM using $T_{1 / 2}$ & ratio \\
\hline 4 & 8378 & 12514 & 1.494 \\
\hline 6 & 37610 & 54453 & 1.448 \\
\hline 8 & 112249 & 161924 & 1.443 \\
\hline 10 & 264606 & 382459 & 1.445 \\
\hline
\end{tabular}

Acknowledgments The authors would like to thank Dr. J. L. Li for helpful discussions. The first and second authors gratefully acknowledge the financial support provided by NSFC (grant 11771137). The research of the third author is supported by a Singapore 


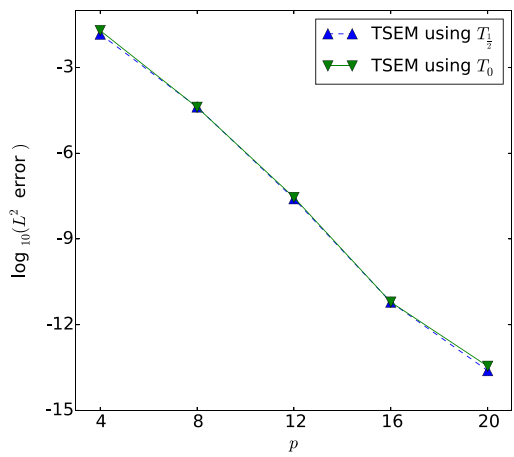

(a) smooth solution

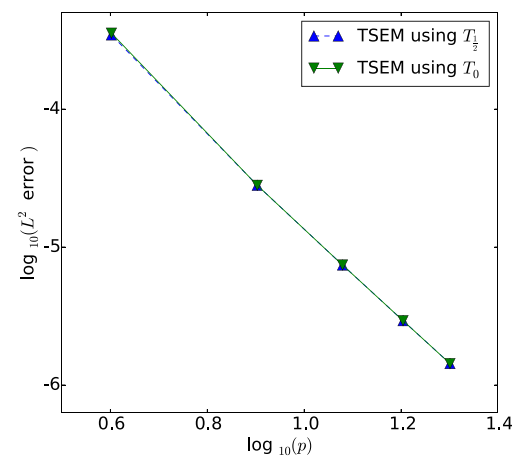

(c) non-smooth solution (4.22b)

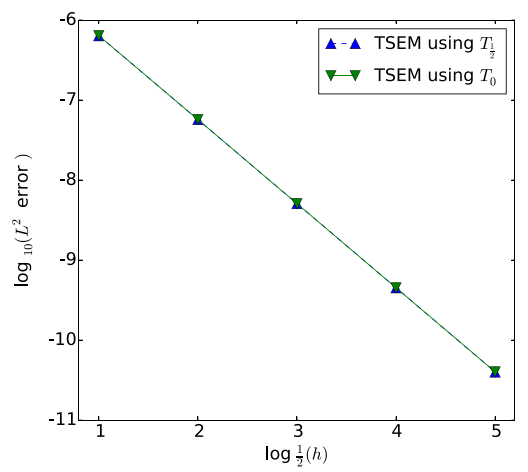

(e) non-smooth solution (4.22a)

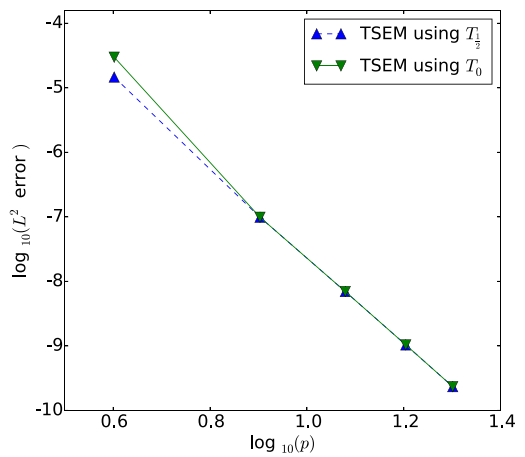

(b) non-smooth solution (4.22a)

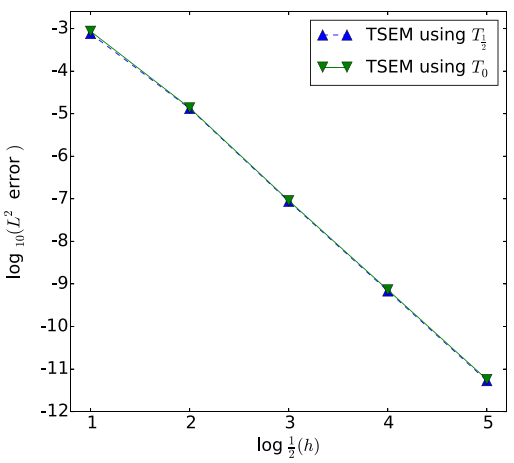

(d) smooth solution

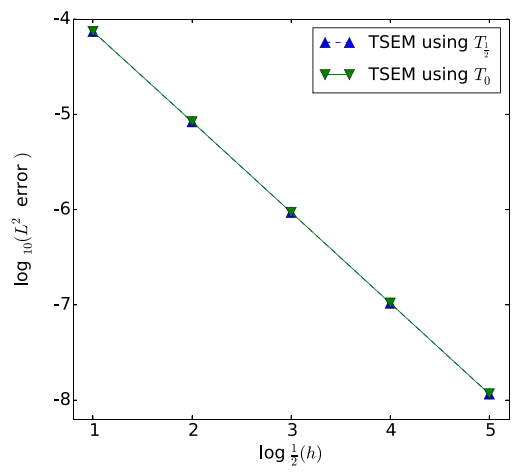

(f) non-smooth solution (4.22b)

Figure 8: Numerical errors and convergence rates of TSEMs on mixed (triangular and rectangular) meshes ((a), (b), (c): errors against $p ;(d),(e),(f)$ : errors against $h)$.

MOE AcRF Tier 2 Grant (MOE2017-T2-2-144), and Singapore MOE AcRF Tier 1 Grant (RG 27/15). The fourth author is partially supported by NSFC (91430107, 11171104 and 11771138) and the Construct Program of the Key Discipline in Hunan Province. 


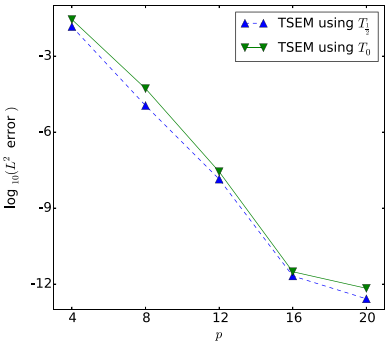

(a) smooth solution

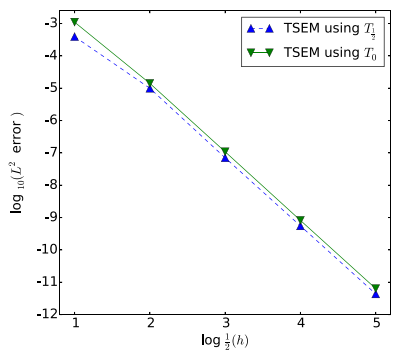

(d) smooth solution

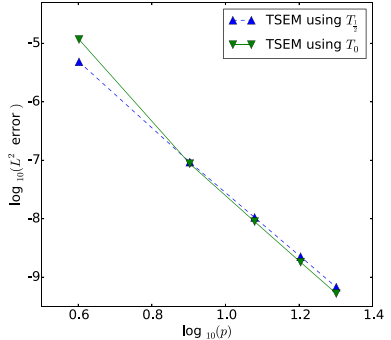

(b) non-smooth solution (4.22a)

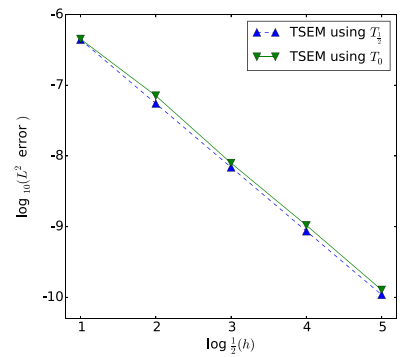

(e) non-smooth solution (4.22a)

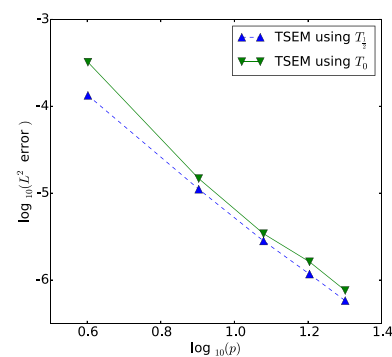

(c) non-smooth solution (4.22b)

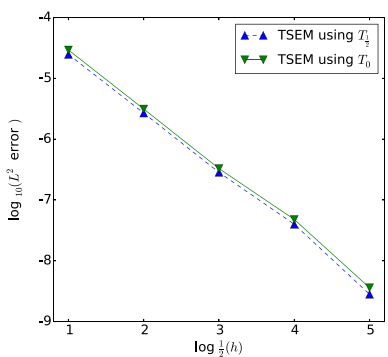

(f) non-smooth solution (4.22b)

Figure 9: Numerical errors and convergence rates of TSEMs on unstructured triangular meshes $((a)$, (b), (c): errors against $p$; (d), (e), (f): errors against $h$ ).

\section{References}

[1] R. A. Adams, Sobolev Spaces, Acadmic Press, New York, 1975.

[2] C. Bernardi, Y. Maday And F. Rapetti, Basics and some applications of the mortar element method, GAMM-Mitt., 28(2) (2005), pp. 97-123.

[3] D. Braess and C. Schwab, Approximation on simplices with respect to weighted sobolev norms, J. Approx. Theory, 103(2) (2000), pp. 329-337.

[4] C. Canuto, M. Y. Hussaini, A. Quarteroni and T. A. Zang, Spectral Methods: Evolution to Complex Geometries and Applications to Fluid Dynamics, Springer, Berlin, 2006.

[5] L. Z. Chen, J. Shen And C. J. Xu, A unstructured nodal spectral-element method for the navierstokes equations, Commun. Comput. Phys., 12(01) (2012), pp. 315-336.

[6] Q. CHEN AND I. BABUŠKA, Approximate optimal points for polynomial interpolation of real functions in an interval and in a triangle, Comput. Methods in Appl. Mech. Eng., 128(3-4) (1995), pp. 405-417.

[7] P. G. Ciarlet, The Finite Element Method for Elliptic Problems, SIAM, Philadelphia, PA. Reprint of the 1978 original North-Holland, Amsterdam, 2002.

[8] M. O. Deville, P. F. Fischer, And E. H. Mund, High-Order Methods for Incompressible Fluid Flow, Cambridge University Press, Cambridge, 2002.

[9] M. Dubiner, Spectral methods on triangles and other domains, J. Sci. Comput., 6(4) (1991), pp. 345-390.

[10] M. Duffy, Quadrature over a pyramid or cube of integrands with a singularity at a vertex, SIAM J. Numer. Anal., 19(6) (1982), pp. 1260-1262.

[11] J. Hesthaven, From electrostatics to almost optimal nodal sets for polynomial interpolation in 
a simplex, SIAM J. Numer. Anal., 35(2) (1998), pp. 655-676.

[12] J. S. Hesthaven and T. Warburton, Nodal Discontinuous Galerkin Methods: Algorithms, Analysis and Applications, Springer Science \& Business Media, 2007.

[13] G. E. Karniadakis, AND S. J. Sherwin, Spectral $/ h p$ Element Methods for Computational Fluid Dynamics, Oxford University Press, New York, 2nd Ed., 2005.

[14] D. A. Kopriva, Implementing Spectral Methods for Partial Differential Equations, Springer, Berlin, 2009.

[15] H. Li AND Z. M. Zhang, Efficient spectral and spectral element methods for eigenvalue problems of Schrodinger equations with an inverse square potential, SIAM J. Sci. Comput., 39(1) (2017), pp. A114-AA140.

[16] H. Li AND L. L. WANG, A spectral method on tetrahedra using rational basis functions, Int. J. Numer. Anal. Model., 7(2) (2010), pp. 330-355.

[17] J. Li, H. MA, L. L. Wang and H. Wu, Spectral element methods on hybrid triangular and quadrilateral meshes, Int. J. Numer. Anal. Model., 15(12) (2018), pp. 111-133.

[18] Y. Li, L. L. WANG, H. Li AND H. MA, A new spectral method on triangles, Lecture Notes in Computational Sciences and Engineering, Springer, 76 (2011), pp. 237-246.

[19] R. G. OwENs, Spectral approximations on the triangle, the Royal Society of London A: Mathematical, Physical and Engineering Sciences, the Royal Society,454 (1998), pp. 857-872.

[20] R. PAsquetti And F. RapetTi, Spectral element methods on unstructured meshes: comparisons and recent advances, J. Sci. Comput., 27(1-3) (2006), pp. 377-387.

[21] R. PASQuetTI AND F. RAPETTI, Spectral element methods on unstructured meshes: which interpolation points?, Numer. Algor., 55(2-3) (2010), pp. 349-366.

[22] T. PATERA, A spectral element method for fluid dynamics: laminar flow in a channel expansion, J. Comput. Phys., 54(3) (1984), pp. 468-488.

[23] M. SAmson, H. Li AND L. L. WANG, A new triangular spectral element method I: implementation and analysis on a triangle, Numer. Algor., 64(3) (2013), pp. 519-547.

[24] J. Shen, L. L. WANG AND H. LI, A triangular spectral element method using fully tensorial rational basis functions, SIAM J. Numer. Anal., 47(3) (2009), pp. 1619-1650.

[25] J. Shen, T. TAng and L. L. Wang, Spectral Methods Algorithms, Analysis and Applications, Springer-Verlag, Berlin Heidelberg, (41) 2011.

[26] S. J. SHERWIN AND G. E. KARNIADAKIS, A new triangular and tetrahedral basis for high-order $(h p)$ finite element methods, Internat. J. Numer. Methods Engrg., 38(22) (1995), pp. 3775-3802.

[27] S. J. Sherwin AND G. E. KARNiAdakis, A triangular spectral element method; applications to the incompressible navier-stokes equations, Comput. Methods Appl. Mech. Engrg., 123 (1995), pp. 189-229.

[28] M. TAYloR, B. Wingate and R. Vincent, An algorithm for computing Fekete points in the triangle, SIAM J. Numer. Anal., 38(5) (2000), pp. 1707-1720. 